\title{
SLC27A5 deficiency activates NRF2/TXNRD1 pathway by increased lipid peroxidation in HCC
}

\author{
Qingzhu Gao ${ }^{1}$ Guiji Zhang ${ }^{1}$ Y Yaqiu Zheng ${ }^{1}$ - Yi Yang ${ }^{1}$. Chang Chen ${ }^{2}$ - Jie Xia ${ }^{1}$ Li Liang ${ }^{1}$. Chong Lei ${ }^{1}$ - Yuan Hu ${ }^{1}$. \\ Xuefei $\mathrm{Cai}^{1} \cdot$ Wenlu Zhang $^{1} \cdot$ Hua Tang ${ }^{1} \cdot$ Yaxi Chen ${ }^{1} \cdot$ Ailong Huang ${ }^{1} \cdot$ Kai Wang $\mathbb{D}^{1} \cdot \mathrm{Ni}^{1}$ Tang $\mathbb{D}^{1}$
}

Received: 21 May 2019 / Revised: 10 July 2019 / Accepted: 17 July 2019 / Published online: 31 July 2019

(c) The Author(s), under exclusive licence to ADMC Associazione Differenziamento e Morte Cellulare 2019

\begin{abstract}
Solute carrier family 27 member 5 (SLC27A5/FATP5) is involved in fatty acid transport and bile acid metabolism; however, little is known about its role in human diseases. Here, we first show that SLC27A5 expression is downregulated in hepatocellular carcinoma (HCC) by DNA hypermethylation, and reduced SCL27A5 expression contributes to tumor progression and poor prognosis. Both gain- and loss-of-function studies demonstrated that SLC27A5 has an antiproliferative effect on HCC cells in vitro and in vivo. Knockout of SLC27A5 increases polyunsaturated lipids, leading to increased $\mathrm{NADP}^{+} / \mathrm{NADPH}$ ratio, ROS production as well as lipid peroxidation and the subsequent accumulation of 4-hydroxy-2nonenal (4-HNE) in hepatoma cells. Mass spectrometry analysis found that 4-HNE directly modifies cysteine residues (Cys513, 518) on KEAP1, thus leading KEAP1/NRF2 pathway activation and increases the expression levels of NRF2 target genes, such as TXNRD1. Further, SLC27A5 expression negatively correlates with TXNRD1 expression in hepatoma cells and clinical HCC samples, and blockade of NRF2/TXNRD1 using genetic approaches or inhibitors sensitizes SLC27A5-deficient hepatoma cells to sorafenib treatment. Collectively, we demonstrated that SLC27A5 acts as a novel tumor suppressor by suppressing TXNRD1 expression via the KEAP1/NRF2 pathway in HCC. Combination therapy of sorafenib and NRF2/TXNRD1 inhibitors may be a promising strategy in personalized HCC treatment.
\end{abstract}

These authors contributed equally: Qingzhu Gao, Guiji Zhang, Yaqiu Zheng, Yi Yang

\section{Edited by R.A. Knight}

Supplementary information The online version of this article (https:// doi.org/10.1038/s41418-019-0399-1) contains supplementary material, which is available to authorized users.

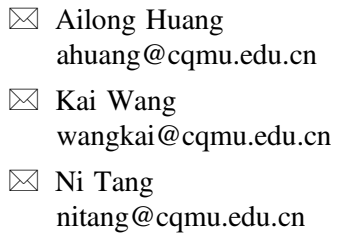

1 Key Laboratory of Molecular Biology for Infectious Diseases (Ministry of Education), Institute for Viral Hepatitis, Department of Infectious Diseases, The Second Affiliated Hospital, Chongqing Medical University, Chongqing, China

2 Institute of Life Sciences, Chongqing Medical University, Chongqing, China

\section{Introduction}

Metabolic reprogramming has been considered as a new hallmark of cancer [1]. Aerobic glycolysis, also called the "Warburg effect", supports various biosynthetic pathways and the metabolic requirements of tumor cell proliferation [2]. Besides altered glucose metabolism, changes in lipid metabolism also contribute to different aspects of tumorigenesis. Increased de novo fatty acid (FA) biosynthesis is a hallmark of many tumors, including hepatocellular carcinoma (HCC) [3]. In addition to de novo lipogenesis, FAs can be exogenously derived as dietary FAs, which are actively transported and uptake into cells mainly by FA transport proteins (FATPs) and the scavenger receptor CD36 [4]. The solute carrier family 27 member 5 gene (SLC27A5), a member of the SLC27A gene family, encodes FA transport protein 5 (FATP5), which is exclusively expressed in the liver. It functions in FA transport and bile acid metabolism [5]. In Slc27a5-knockout mice, long-chain fatty acid uptake is significantly reduced, while de novo synthesis is increased in hepatocytes [6]. These mice failed to gain weight on a high-fat diet because of reduced food 
intake and an increased metabolic rate [7]. Polymorphism in the regulatory region of the $S L C 27 A 5$ promoter $(-1324 \mathrm{G}$ $>$ A) has been reported to be associated with hepatic injury, insulin resistance, and dyslipidemia [8]. However, little is known about the role of SLC27A5 in human diseases, especially, in HCC tumorigenesis.

Deregulated redox homeostasis is another hallmark of cancer cells. Compared with normal cells, cancer cells exhibit persistently high levels of reactive oxygen species (ROS), as a consequence of oncogenic transformation, uncontrolled proliferation, and high metabolic rate [9], whereas excessive amounts of ROS induce oxidative modification of cellular proteins and promote severe cellular damage and cell death [10]. Parallel to the production of ROS, cancer cells have evolved efficient antioxidative machinery to limit ROS accumulation [11]. Glutathione and thioredoxin (TXN) play key roles in redox homeostasis by either direct neutralization of ROS or by indirect recycling small-molecule antioxidants [12]. The TXN antioxidant pathway, comprising NADPH, TXN reductase, and TXN, is activated in cancer [13]. Thioredoxin reductase 1 (TXNRD1), one form of TXN reductase, is upregulated in multiple cancers, including HCC and recognized an unfavorable prognostic factor [14]. Enhanced expression of TXNRD1 is associated with advanced tumor progression and metastasis [15] and confers chemotherapeutic resistance [16].

Here, the role of SLC27A5 in human hepatic carcinogenesis was explored. We found that the expression of SLC27A5 is downregulated in HCC cell lines and tumor tissues. Overexpression of SLC27A5 suppresses the growth of hepatoma cells in vitro and in vivo. Mechanistically, we demonstrated that loss of SLC27A5 elevates TXNRD1 expression via activation of the KEAP1/NRF2 pathway. Further, blockade of NRF2/TXNRD1 sensitizes SLC27A5-deficient hepatoma cells to sorafenib treatment. Collectively, our results implicate that SLC27A5 acts as a tumor suppressor in HCC and combination therapy of sorafenib and NRF2/TXNRD1 inhibitors may be a potential strategy in HCC treatment.

\section{Materials and methods}

\section{Cell culture}

Human HCC cell lines PLC/PRF/5, HepG2 were obtained from the American Type Culture Collection (ATCC, VA, USA); MHCC-97H, SK-Hep1, Hep3B, and Huh7 from the Cell Bank of the Chinese Academy of Sciences (Shanghai, China). One immortalized liver cell line (MIHA) was kindly gifted by Professor Ben C.B. Ko (The Hong Kong Polytechnic University). Cells were maintained in Dulbecco's modified Eagle's medium (DMEM; Hyclone, Logan, UT, USA) supplemented with $10 \%$ fetal bovine serum (FBS; Gibco,
Rockville, MD, USA), $100 \mathrm{mg} / \mathrm{mL}$ of streptomycin, and 100 unit $/ \mathrm{mL}$ of penicillin at $37^{\circ} \mathrm{C}$ in $5 \% \mathrm{CO}_{2}$. All cells were routinely tested for absence of mycoplasma contamination.

\section{Adenoviruses and reporter plasmids}

The full-length cDNA of SLC27A5 (NM_012254.2) was amplified from plasmid EX-W0248-M02 (GeneCopoeia, Guangzhou, China) and inserted into the Kpn I and Hind III sites of the shuttle vector pAdTrack-TO4 (from Dr T-C He, University of Chicago, USA). The adenoviral recombinant pAd-SLC27A5 was generated using the AdEasy system [17]. The expression vector of human Nrf2 was made by subcloning PCR-amplified inserts into pcDNA-5Myc vector (a gift from from prof. Wei Lv, Shanghai Institute of Nutrition and Health, Chinese Academy of Sciences). The expression vector of human Keap1 was made by subcloning PCRamplified inserts into pSEB-3Flag vector (a gift from Dr T-C He, University of Chicago, USA). The Flag-tagged Keap1 was subcloned into the shuttle vector pAdTrack-TO4 and generated the adenoviral recombinant Ad-Keap1-3Flag using the AdEasy system. An analogous adenovirus expressing only GFP (AdGFP) was used as control. The Cys to Ala or the Cys to Ser mutant of Keap1 (C151, C513, and C518) was constructed by site-directed mutagenesis, with wild-type Keap13Flag plasmid as a template. The authenticity of these constructs was confirmed by DNA sequencing.

\section{RNA interference}

The small double-strand hairpin shRNA for TXNRD1 were designed and annealed into the Hpa I/ Xho I sites of pLL3.7 lentivirus vector (kindly provided by Prof. Bing Sun, the Institute Pasteur of Shanghai, Chinese Academy of Sciences). Lentivirus was generated as reported previously [18]. Briefly, pLL3.7 vectors encompassing shRNAs targeting TXNRD1 (shTXNRD1), or a scrambled control were transfected into HCC cell lines.

\section{CRISPR/Cas9-mediated knockout of SLC27A5}

The CRISPR/Cas9 plasmids lentiCRISPR v2, pMD2.G, and psPAX2 were kindly provided by Prof. Ding Xue from the Tsinghua University (Beijing, China). For SLC27A5 knockout (KO) cell line development, single-guide RNAs (sgRNAs) were cloned into the lentiCRISPR-V2 vector [19]. Lentivirus was generated by co-transfecting HEK293T cells with lentiCRISPR v2, envelop plasmid pMD2.G, and packaging plasmid psPAX2 using Lipofectamine 2000 (Invitrogen, Carlsbad, CA, USA), following the manufacturer's protocol. HepG2 cells were then infected with filtered lentiviral supernatant and selected with $0.5 \mathrm{mg} / \mathrm{mL}$ of puromycin (ab141453, Abcam, Cambridge, MA, USA). The 
single-cell HCC clones stably expressing sgRNA were propagated and validated by immunoblotting and DNA sequencing. Sequences of all sgRNAs and shRNAs are listed in Table S1.

\section{Luciferase reporter assay}

PLC/PRF/5 cells were transfected with $3 \mu \mathrm{g}$ of pGL3-ARELuc (provided by Prof. Yiguo Zhang from Chongqing University) and $300 \mathrm{ng} / \mathrm{well}$ pRL-TK (an internal control) using Lipofectamine 2000. After $24 \mathrm{~h}$, cells were infected with adenoviruses expressing AdSLC27A5 or vector control (AdGFP). At $36 \mathrm{~h}$ post infection, cells were harvested and assayed for luciferase activity using the Dual Luciferase Assay Kit (Promega, Madison, WI, USA). All experiments were performed at least three times and expressed as mean \pm standard deviation (SD).

\section{RNA extraction, reverse transcription PCR, and quantitative real-time PCR}

Total RNA was extracted from clinical specimens and HCC cell lines using TRIzol reagent (Invitrogen, Rockville, MD) according to the manufacturer's instructions. Purified RNA samples were reverse-transcribed into cDNA using the Moloney murine leukemia virus reverse transcriptase (A3500, Promega, Madison, USA). Quantitative Real-Time PCR analysis of target genes was performed using the SYBR Green qPCR Master Mix (Bio-Rad, Hercules, CA, USA) with specific primers (Table S1). Relative expression was calculated as a ratio of the expression of the specific transcript to that of glyceraldehyde 3-phosphate dehydrogenase (GAPDH). Each sample was analyzed in triplicate.

\section{Western blotting}

Protein lysates of cells or liver samples were extracted by cell lysis buffer (Beyotime Biotechnology, Jiangsu, China) containing $1 \mathrm{mM}$ phenylmethanesulfonyl fluoride (Beyotime). Equal volumes of protein samples were separated by $10 \%$ SDS/PAGE and electro-transferred to PVDF membranes (Millipore, Billerica, MA, USA). The immunoblots were probed with the indicated antibodies. Proteins bands were visualized with Super Signal West Pico Chemiluminescent substrate Kits (Millipore).

\section{Immunohistological staining}

Liver tissue samples were fixed in $4 \%$ paraformaldehyde and embedded in paraffin according to standard procedures. Sections were incubated with the indicated primary antibodies overnight at $4{ }^{\circ} \mathrm{C}$. Subsequently, the slides were incubated with a secondary anti-rabbit or anti-mouse IgG (ZSGB-BIO,
Beijing, China) and visualized using 3,3'-diaminobenzidine (ZSGB-BIO). Stained slides were scanned with a Pannoramic Scan 250 Flash or MIDI system and images acquired using Pannoramic Viewer 1.15.2 (3DHistech, Budapest, Hungary). The mean staining intensity was analyzed by Image-Pro Plus. 6.0 (Media Cybernetics, Inc, Bethesda, MD, USA).

\section{Cell apoptosis assay by flow cytometry}

Cells were cultured in medium containing 5\% serum for $48 \mathrm{~h}$ before trypsinization and subsequently resuspended in APC Annexin V buffer (BD Biosciences, CA, USA) and DAPI (BD Biosciences) for staining according to the manufacturer's instructions. The percentage of cell apoptosis for each sample was analyzed with flow cytometry.

\section{Cell proliferation and colony formation assays}

Cell counting, 5-ethynyl-2'-deoxyuridine (EdU) incorporation, and colony formation were used to measure cell proliferation rates. The IncuCyte ZOOM Live-Cell Imaging system (Essen BioScience, Ann Arbor, MI, USA) was used for monitoring cell proliferation. Cells were seeded in 96well plates at $3 \times 10^{3}$ cells/well and were cultured for 6 days. The plate was scanned and phase-contrast images were acquired in real time every $24 \mathrm{~h}$ post treatment, and quantified time-lapse curves were generated by IncuCyte ZOOM software. For EdU incorporation assays, $5 \times 10^{4}$ cells per well were plated into six-well culture plates. EdU labeling and fluorescence measurements were performed $24 \mathrm{~h}$ after plating according to the manufacturer's instructions (Ribobio, Guangzhou, China). For colony formation assays, $2 \times 10^{3}$ cells per well were seeded in six-well plate with DMEM containing 10\% serum for 2 weeks. Colonies were stained with $0.004 \%$ crystal violet, photographed, and counted in triplicate.

\section{Immunofluorescence}

NRF2 staining was detected with a polyclonal rabbit antiNRF2 (Abcam, ab62352), and specific signals were visualized with Alexa Fluor 488 or 594 secondary antibody (Invitrogen). Nuclear staining was achieved using $1 \mathrm{ug} / \mathrm{mL}$ DAPI (10236276001, Roche Diagnostics GmbH, Mannheim, Germany) for $3 \mathrm{~min}$. Stained sections were analyzed by a laser scanning confocal microscope (Leica TCS SP8, Solms, Germany).

\section{Nontargeted lipidomics}

Briefly, frozen hepatoma cells were first suspended with a chloroform:methanol water mixture $(2: 1, \mathrm{v} / \mathrm{v})$. After phase 
breaking, the lower-layer fluid was collected and blow-dried by vacuum concentration. Samples were dissolved with isopropanol and filtrated with $0.22 \mu \mathrm{m}$ membrane prior to analysis. Quality control samples were prepared by pooling equal amounts of lipid extracts from every sample. Chromatographic separations were performed on Thermo Ultimate 3000 system equipped with an ACQUITY UPLC ${ }^{\circ}$ BEH C18 $(100 \times 2.1 \mathrm{~mm}, 1.7 \mu \mathrm{m}$, Waters Corp., Milford, MA, USA) column. Gradient elution of samples was performed with water $(0.1 \%$ formic acid $+10 \mathrm{mM}$ ammonium formate $)$ and isopropanol:acetonitrile $=2: 5(0.1 \%$ formic acid $+10 \mathrm{mM}$ ammonium formate). The flow rate was maintained at $0.3 \mathrm{ml} / \mathrm{min}$. The ESI-MSn experiments were operated on the Thermo Q Exactive Focus mass spectrometer in the electrospray ionization-positive and ionizationnegative modes with the mass range set at $\mathrm{m} / \mathrm{z}$ 150-2000.

\section{Fatty acid oxidation assays}

Oxygen consumption rate (OCR) of PLC/PRF/5 cells and HepG2 cells were measured using the Seahorse Bioscience Extracellular Flux Analyzer (XFe24; Seahorse Bioscience, North Billerica, MA, USA). Briefly, cells were plated at 40,000 cells/well with $250 \mu \mathrm{L}$ culture medium in a 24 -well cell culture plate (Seahorse Bioscience). $24 \mathrm{~h}$ prior to the assay, the growth medium was replaced with substratelimited DMEM (catalog A14430-01, Life technologies, Carlsbad, CA, USA) supplemented with $0.5 \mathrm{mM}$ glucose (Sigma-Aldrich, St. Louis, MO, USA), $1 \mathrm{mM}$ glutamine (Life technologies), $0.5 \mathrm{mM}$ carnitine (Sigma-Aldrich), $1 \%$ FBS and incubated at $37{ }^{\circ} \mathrm{C}, 5 \% \mathrm{CO}_{2}$ overnight. On the day of assay, the FAO assay buffer $(111 \mathrm{mM} \mathrm{NaCl}, 4.7 \mathrm{mM}$ $\mathrm{KCl}, 1.25 \mathrm{mM} \mathrm{CaCl}_{2}, 2 \mathrm{mM} \mathrm{MgSO}_{4}, 1.2 \mathrm{mM} \mathrm{NaH}_{2} \mathrm{PO}_{4}, 2.5$ $\mathrm{mM}$ glucose, $0.5 \mathrm{mM}$ carnitine, and $5 \mathrm{mM}$ HEPES, $\mathrm{pH} 7.4$ with $\mathrm{NaOH}$ ) was exchanged and incubated in a non- $\mathrm{CO}_{2}$ incubator for $45 \mathrm{~min}$ at $37^{\circ} \mathrm{C}$. To examine free fatty acid oxidation (FAO), BSA conjugated palmitate, or BSA vehicle (Seahorse Bioscience) was added to a final concentration of $175 \mu \mathrm{M}$. The hydrated XF24 sensor cartridge was loaded Seahorse XF Cell Mito Stress Test compounds (final concentrations: $1 \mu \mathrm{g} / \mathrm{mL}$ oligomycin, $1.5 \mu \mathrm{M}$ FCCP, and $0.5 \mu \mathrm{M}$ rotenone $/ 0.5 \mu \mathrm{M}$ antimycin A). Then the OCR of cells treated with palmitate-conjugated BSA or BSA vehicle alone were measured following the manufacturer's manual.

\section{ROS and NADPH measurements}

Cells or freshly prepared frozen liver sections were incubated with $5 \mu \mathrm{mol} / \mathrm{L}$ fluorescent dye dihydroethidium (C1793768, DHE, CellROX Orange, Life Technologies) at $37^{\circ} \mathrm{C}$ for $30 \mathrm{~min}$, and then washed with cold PBS. Intracellular ROS levels were analyzed by fluorescence microscope (Leica TCS SP8, Solms, Germany). Red fluorescence intensity was quantified by using ImageJ software. Intracellular $\mathrm{NADP}^{+} / \mathrm{NADPH}$ ratios in $\mathrm{HCC}$ cells were measured using the NADP/NADPH Quantification Kit (ab65349, Abcam) according to the manufacturer's instructions. Briefly, $4 \times 10^{6}$ cells were trypsinized and subjected to extraction by two freeze/thaw cycles using 800 $\mu \mathrm{l}$ of the Extraction Buffer. Signal Intensities for NADP and NADPH were examined by OD measurements at $450 \mathrm{~nm}$. The $\mathrm{NADP}^{+} / \mathrm{NADPH}$ ratio was calculated as: (intensity of unheated samples-intensity of heated samples)/(intensity of heated samples).

\section{TUNEL staining}

Sections of tumor tissue or liver tissue from nude mice were subjected to deparaffinization, rehydration. Sections were then subjected to cell death detection according to the manufacturer's instructions (In Situ Cell Death Detection Kit, POD, Roche Diagnostics), followed by counterstaining with 3,3'-diaminobenzidine. Results were analyzed by counting positively stained cells and dividing by the total cells.

\section{Immunoprecipitation assay}

SLC27A5-OE PLC/PRF/5 cells or SLC27A5-KO HepG2 cells were infected with Flag-tagged Keap1 adenoviruses for $48 \mathrm{~h}$. Cells were resuspended with cell lysis buffer (Beyotime) containing protease inhibitor cocktail (Roche) and precleared supernatants were incubated with monoclonal anti-Flag (MA1-91878, Invitrogen) overnight at $4{ }^{\circ} \mathrm{C}$, followed by $4 \mathrm{~h}$ incubation with protein $\mathrm{A} / \mathrm{G}$ agarose beads. Immunoprecipitated complexes were eluted and subjected to immunoblotting with the indicated antibodies.

\section{Analysis of Keap1 modification by mass spectrometry}

Analysis was performed using an LTQ-Orbitrap Velos (Thermo Scientific) mass spectrometer coupled with a nanoUPLC system (nanoAcquity Ultra Performance LC, Waters). Protein samples were reduced with $5 \mathrm{mM}$ dithiothreitol at $42{ }^{\circ} \mathrm{C}$ for $30 \mathrm{~min}$, then alkylated with $10 \mathrm{mM}$ iodoacetamide in the dark at room temperature for $30 \mathrm{~min}$. Proteins were digested with Trypsin $(10 \mathrm{ng} / \mu \mathrm{L})$ at $37^{\circ} \mathrm{C}$ for $12 \mathrm{~h}$ and resulting peptides were extracted from the gel with extracting buffer (50\% acetonitrile (ACN), $5 \%$ formic acid). Peptides were separated on a self-packed capillary column ( $75 \mu \mathrm{m}$ i.d. $\times 15 \mathrm{~cm}, 3 \mu \mathrm{m} \mathrm{C18}$ reverse-phase resin). Peptides were eluted using a 60 min nonlinear gradient: $4 \% \mathrm{~B}$ for $6 \mathrm{~min}, 4-10 \% \mathrm{~B}$ for $6 \mathrm{~min}, 10-24 \% \mathrm{~B}$ for $30 \mathrm{~min}$, $24-34 \%$ B for $15 \mathrm{~min}, 34-80 \%$ B for $1 \mathrm{~min}, 80 \%$ B for 2 min (Buffer A, $0.1 \%$ formic acid in $\mathrm{ddH}_{2} 0$; Buffer B, 0.1\% 
formic acid and $98 \% \mathrm{AcN}$ in $\mathrm{ddH}_{2} \mathrm{O}$; flow rate, $\sim 300 \mathrm{~nL} /$ $\min )$. The MS survey scans were acquired at a resolution of 30,000 , and target values of $1,000,000$ ions over a mass range from 300 to $1600 \mathrm{~m} / \mathrm{z}$. Collision-induced dissociation was performed on the twenty most abundant ions per full MS scan using an isolation width of 2.0 Da. All fragmented precursor ions were actively excluded from repeated MS/ MS analysis for $30 \mathrm{~s}$. The raw files were searched with the Maxquant (v1.5.3.30) against the Swiss-Prot reviewed human database (2017-02). Full cleavage by trypsin was set including two miscleavages. Identifications satisfying the criteria, FDR $\leq 1 \%$ at both peptide and protein levels, were considered.

\section{Methylation Analyses of the SLC27A5 Promoter}

Genomic DNA extraction from $20 \mathrm{mg}$ of human liver tissue was performed using The Wizard Genomic DNA Purification Kit (Promega, Madison, WI, A1120). Bisulfite conversion of Genomic DNA was performed according to the manufacturer's instructions (EZ DNA MethylationGold $^{\mathrm{TM}}$ Kit D5005, Zymo Research, CA, USA). The specific primers for SLC27A5 are listed in Table S1. For quantitative methylation analysis of SLC27A5 CpG island promoter region, bisulfite sequencing PCR (BSP) was carried out by subcloning the BSP products into pMD19-T vector (Takara, Dalian, China), and then transformed into Escherichia coli DH5 $\alpha$. At least ten successful plasmid clones were chosen for sequencing. The methylation ratio was calculated as the fraction of methylated $\mathrm{CpG}$ dinucleotides of the total $\mathrm{CpG}$ sites analyzed.

\section{Mouse embryo fibroblast (MEF) cell isolation}

Wild-type and NRF2 KO mice were kindly provided by Dr Hongting Zheng from the Army Medical University [20]. The mouse embryo fibroblast (MEF) cells were isolated according to the MEF feeder cell preparation [21]. Briefly, mice were killed at day 12.5 of gestation. The embryos were dissociated and trypsinized to produce single-cell suspensions.

\section{Antibodies}

Anti-SLC27A5 (1:200\&1:100, BM2177,BOSTER, China), Anti- $\beta$-actin (1:2000, BL005B, Biosharp, China), AntiTXNRD1 (1:100, GTX108727, Gene Tex, USA), AntiNRF2 (1:1000\&1:200, Ab62352, Abcam, USA), Anti-Lamin B1 (1:10000, AP6001, Bioworld Technology, USA), Anti- $\beta$ Tublin (1:5000, 66240-1-Ig, Proteintech Group, USA), AntiFlag (1:5000\&1:300, MA1-91878, Invitrogen, USA), AntiMyc (1:1000, 2278S, Cell Signaling Technology, USA), Anti-4-HNE (1:3000, Ab46545, Abcam, USA), AntiGAPDH (1:2000, AG019, Beyotime, China), Anti-Ki67
(1:20, PA516785, Thermo Fisher Scientific, USA), AntiBax (1:1000, RLT0456, Ruiying Biological, China), AntiBcl2 (1:1000 RLM3041, Ruiying Biological, China), and Anti-PARP (1:1000, 9542, Cell Signaling Technology, USA).

\section{Chemicals and reagents}

4-Hydroxynonenal (4-HNE) reagent was purchased from Cayman Chemical (Ann Arbor, MI). tBHQ was obtained from Sigma-Aldrich (112941). The MDA assay kit, Nuclear and Cytoplasmic Protein Extraction Kit were purchased from Beyotime Institute of Biotechnology (Shanghai, China).

\section{Patient samples}

HCC tissues and paired nontumorous tissues were obtained from 40 patients who underwent surgery at the Second Affiliated Hospital of Chongqing Medical University between 2014 and 2017, with the approval of the Institutional Review Board of Chongqing Medical University. Patients provided informed consent and had not received chemotherapy or radiation therapy before surgery. All specimens were frozen immediately after surgery and stored in liquid nitrogen until use.

\section{Clinicopathological correlation analysis and survival analysis}

Clinicopathological features of HCC patients were analyzed by $\chi^{2}$ analysis using SPSS 17.0 software (IBM Corp, Armonk, NY, USA). Survival data of 180 HCC patients were obtained from The Cancer Genome Atlas (TCGA) database. Survival analysis was conducted by the Kaplan-Meier method followed by the log-rank test.

\section{Animal model and treatment}

Animal experiments were conducted using 4-6-week-old $\mathrm{BALB} / \mathrm{c}$ nude mice (male, 20-25 g), mice were provided by the Laboratory Animal Center of Chongqing Medical University (SCXK (YU) 2017-0001). For the xenograft implantation model, a total of 36 nude mice were randomly divided into six groups, $2 \times 10^{6}$ cells were subcutaneously injected into the flanks of the mice. Treatment was initiated when the tumors were $50-100 \mathrm{~mm}^{3}$ in size. Tumor-bearing mice were intraperitoneally injected with auranofin $(10 \mathrm{mg} / \mathrm{kg} /$ day, 6.8 $\mathrm{mg} / \mathrm{mL}$ in $5 \%$ dimethyl sulfoxide (DMSO)), sorafenib (30 $\mathrm{mg} / \mathrm{kg} / \mathrm{day}, 3 \mathrm{mg} / \mathrm{mL}$ in $5 \%$ DMSO), both, or vehicle (5\% DMSO) ( $n=6$ per group) for 14 days. Tumor volume (V) was calculated as: $\mathrm{V}\left[\mathrm{cm}^{3}\right]=($ length $[\mathrm{cm}]) \times($ width $[\mathrm{cm}] \times$ (width $[\mathrm{cm}]$ )/2. After 4 weeks, the mice were killed, and tumor tissues were harvested for histological analysis. 

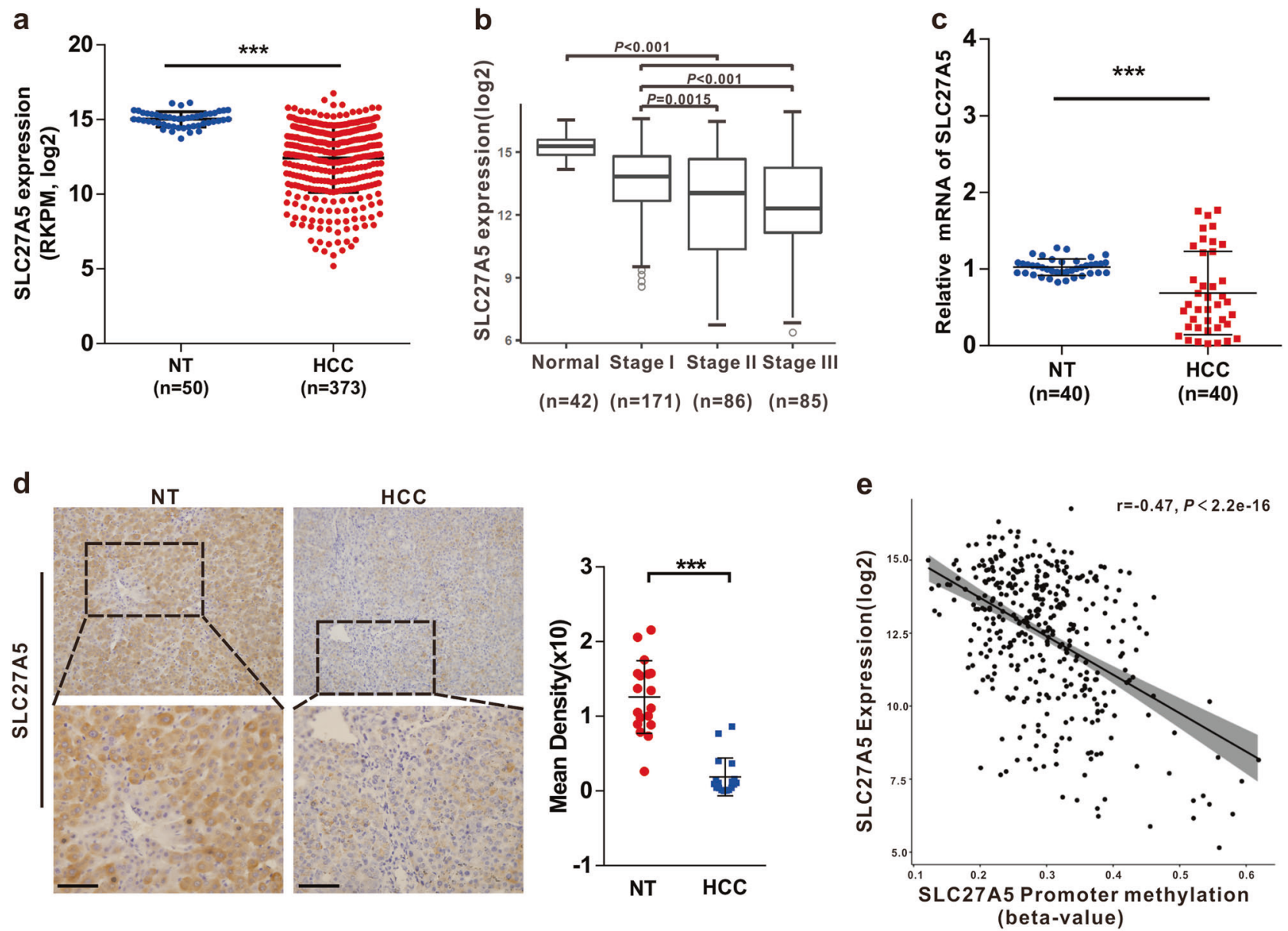

f

\section{Forward primer}

СтGGGCAGGAAGATCCCATAGTGAGGGGAGGGTTCTCTGATAGGCAAGGGATCCCGGGGTGGTGGTTCGGGAGGGGAGGGGAGCCCCT GCTAGGTCCAGGAGCCCAGGTGGGGGTGGGGTGGGGTGGGGGCGGGAGCTGTAGAGTCTGACTTGCGTTCGCGGCCCGCCCTGTCTT CCGCAGGGGAGAAGCCGACCAGTGCGCCGTTGCTGGCGATCCTTCTCTCTACGCCGACTACCTGCTCAAACACATGGTC

Reverse primer

g

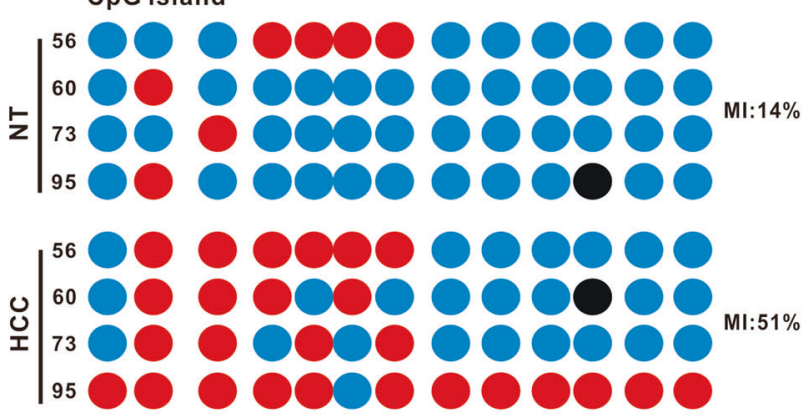

unmethylated $\mathrm{CpG}$ locus

methylated $\mathrm{CpG}$ locus h

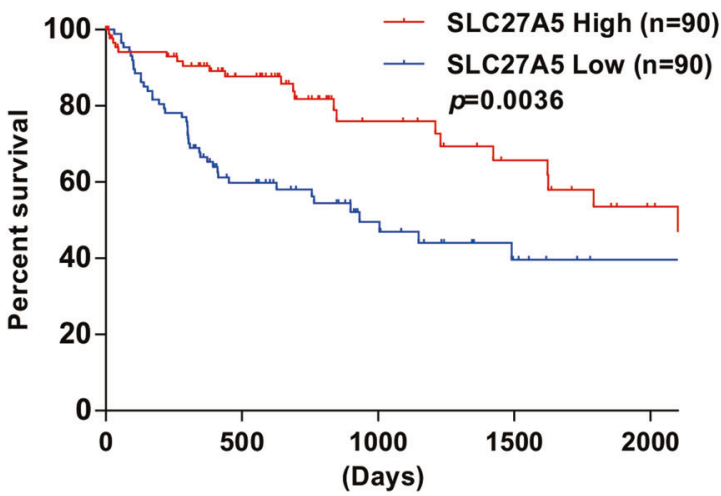


Fig. 1 SLC27A5 expression is generally downregulated in human HCC tissues and correlates with poor patient prognosis. a Expression of SLC27A5 in patients with HCC based on data from TCGA Liver Hepatocellular Carcinoma dataset. RKPM, Reads per kilobase of exon model per million mapped reads. $* * * P<0.001$, two-tailed $t$-test. b Box plots of SLC27A5 expression in normal liver and tumor tissues (Clinical stages I to III) from the TCGA Database. Thirteen patients with stage IV cancer were excluded from the analysis. c SLC27A5 mRNA expression in 40 paired HCC (T) and nontumorous (NT) liver tissues. $* * * P<0.001$, Wilcoxon's signed-rank test. d IHC staining of SLC27A5 in HCC tissues and adjacent nontumor tissues, bar $=50 \mu \mathrm{m}$. Immunostaining intensity was assessed using Image-Pro plus 6.0 software. $* * * P<0.001$, Student's $t$-test. e SLC27A5 expression levels in tumor samples were inversely correlated with the degree of SLC27A5 methylation in TCGA (Pearson correlation coefficient $=$ $-0.47 ; P<0.001)$. f Nucleotide sequence of SLC27A5 that forms a part of $\mathrm{CpG}$ islands around the promoter region. Individual $\mathrm{CpG}$ loci are highlighted in red. g Direct bisulfite sequencing of SLC27A5 promoter region for individual $\mathrm{CpG}$ loci in HCC tissues (T) and adjacent nontumor tissues (NT). MI: methylation index $=$ (number of methylated $\mathrm{CpG}$ loci)/(total number of $\mathrm{CpG}$ loci). h Kaplan-Meier survival analysis of TCGA cohort; HCC patients were divided into two groups on the basis of the SLC27A5 expression level in tumor (top $25 \%$, high; bottom $25 \%$, low)

For the orthotopic implantation model, total number of 24 nude mice were randomly divided into four groups, $4 \times$ $10^{6}$ tumor cells were suspended in a $40 \mu \mathrm{l}$ PBS/Matrigel (356234, BD Biosciences) mixture (1:1 (v/v) ratio) for each nude mice and implanted into the left liver lobes of nude mice. Thirty days after inoculation, tumor-bearing mice were administered sorafenib $(30 \mathrm{mg} / \mathrm{kg} / \mathrm{d})$ or vehicle $(n=6$ per group) for 14 days. At 7 weeks after implantation, mice were killed, and liver tissues were harvested for histological examination. All animal studies were followed by a blind randomized study protocol.

\section{Drug treatments}

For in vitro assays, $50 \mathrm{mM}$ sorafenib (S7397, Selleckchem, USA) dissolved in 100\% DMSO (Sigma, USA), $1 \mathrm{mM}$ brusatol (MB7292, Meilunbio) dissolved in 100\% DMSO and $10 \mathrm{mM}$ auranofin (HY-B1123, MedChemExpress, USA) dissolved in $100 \%$ DMSO were used for stock solution. Cells were treated in the presence of $1 \% \mathrm{FBS}$ DMEM at final 2\% DMSO with: $5 \mu \mathrm{M}$ sorafenib or $60 \mathrm{nM}$ brusatol; $5 \mu \mathrm{M}$ sorafenib or $0.6 \mu \mathrm{M}$ auranofin; or combined therapy; or untreated vehicle control.

\section{Study approval}

For patient samples, the study protocol was approved by the Medical Ethics Committee of Chongqing Medical University. Patients were provided informed consent and had not received radiation or chemotherapy therapy before surgery. All experimental procedures performed on animals were approved by Institutional Animal Care and Use
Table 1 Clinical correlation between mRNA levels of SLC27A5 and clinicopathologic parameters of HCC patients

\begin{tabular}{|c|c|c|c|c|}
\hline \multirow[t]{2}{*}{ Variable } & \multirow[t]{2}{*}{ Frequency $(\%)$} & \multicolumn{2}{|c|}{$\begin{array}{l}\text { SLC27A5 relative } \\
\text { expression }\end{array}$} & \multirow[t]{2}{*}{$P$ value } \\
\hline & & $\begin{array}{l}\text { Low } \\
(n=30)\end{array}$ & $\begin{array}{l}\text { High } \\
(n=10)\end{array}$ & \\
\hline \multicolumn{5}{|l|}{ Sex } \\
\hline Male & $31(77.5)$ & 24 & 7 & \multirow[t]{2}{*}{0.827} \\
\hline Female & $9(22.5)$ & 6 & 3 & \\
\hline \multicolumn{5}{|l|}{ Age } \\
\hline$\leq 55$ & $26(65)$ & 19 & 7 & \multirow[t]{2}{*}{1} \\
\hline$>55$ & $14(35)$ & 11 & 3 & \\
\hline \multicolumn{5}{|l|}{ Tumor size, $\mathrm{cm}$} \\
\hline$>5$ & $21(52.5)$ & 19 & 2 & \multirow[t]{2}{*}{$0.044 *$} \\
\hline$\leq 5$ & $19(47.5)$ & 11 & 8 & \\
\hline \multicolumn{5}{|c|}{ Alpha fetoprotein, $\mathrm{ng} / \mathrm{mL}$} \\
\hline$\leq 100$ & $19(47.5)$ & 14 & 5 & \multirow[t]{2}{*}{1} \\
\hline$>100$ & $21(52.5)$ & 16 & 5 & \\
\hline \multicolumn{5}{|c|}{ Hepatitis B surface antigen } \\
\hline Positive & $32(80)$ & 23 & 9 & \multirow[t]{2}{*}{0.648} \\
\hline Negative & $8(20)$ & 7 & 1 & \\
\hline \multicolumn{5}{|c|}{ Histological differentiation } \\
\hline Well & $12(30)$ & 5 & 7 & \multirow[t]{2}{*}{$0.005 *$} \\
\hline Moderate/poor & $28(70)$ & 25 & 3 & \\
\hline \multicolumn{5}{|c|}{ Intrahepatic metastasis } \\
\hline Absent & $29(72.5)$ & 21 & 8 & \multirow[t]{2}{*}{0.838} \\
\hline Present & $11(27.5)$ & 9 & 2 & \\
\hline \multicolumn{5}{|l|}{ Recurrence } \\
\hline Absent & $18(45)$ & 9 & 9 & \multirow[t]{2}{*}{ 0.003* } \\
\hline Present & $22(55)$ & 21 & 1 & \\
\hline
\end{tabular}

A total of 40 paired HCC tissues with defined clinical data were chosen for this correlation study

$* P<0.05$

Committee at the Chongqing Medical University (license number: 2017011). All mice were maintained under specific pathogen-free conditions in the laboratory animal center of Chongqing Medical University. Animal care and use protocols adhere to National Regulations for the Administration of Laboratory Animal to ensure minimal suffering.

\section{Statistical analysis}

All data were presented as means \pm SD. Sample sizes for relevant experiments were determined by power analyses conducted during experiment planning. Appropriate statistical analyses, Fisher test, or Levene test for equality of variances were performed using GraphPad Prism 5.0 software (GraphPad Software Inc, La Jolla, CA, USA). Statistical significance was determined using one-way ANOVA 
a

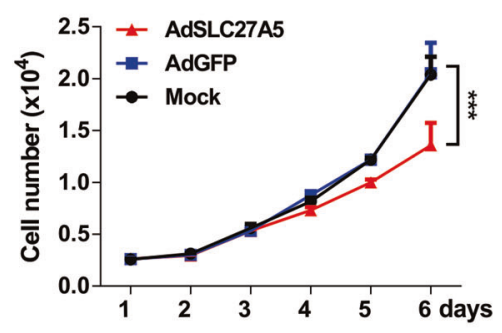

b

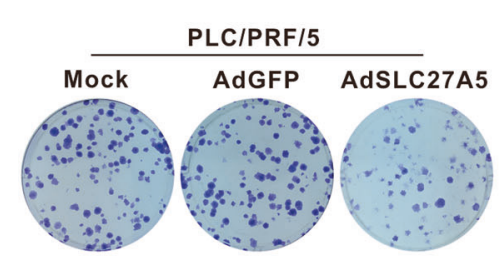

C

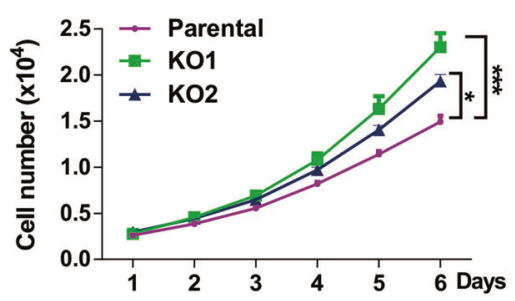

SK-Hep1

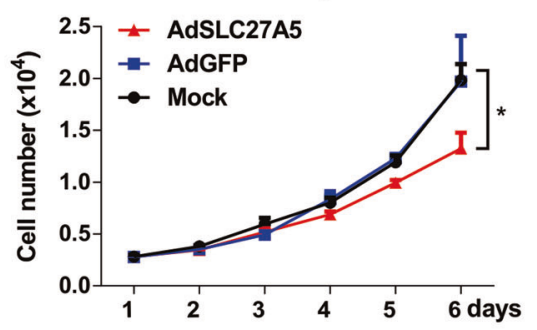

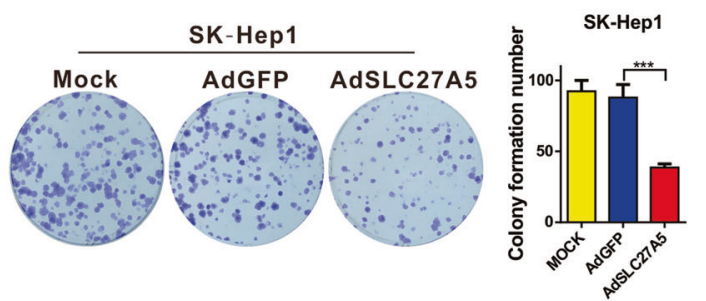

d

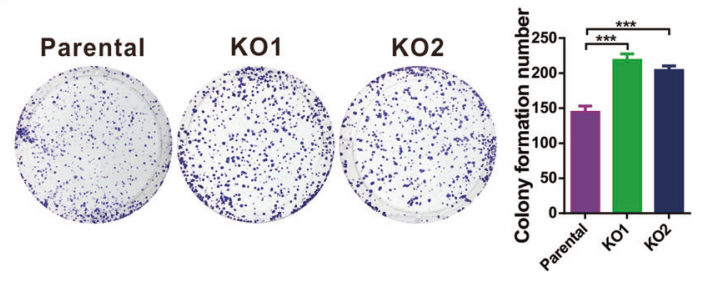

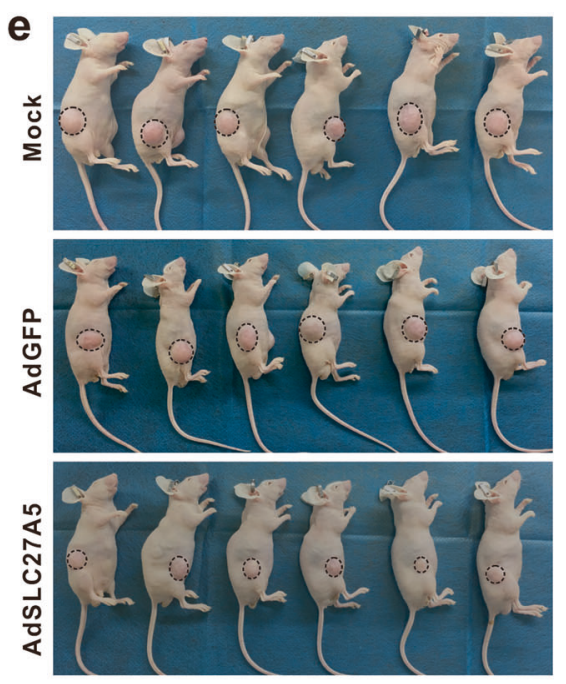
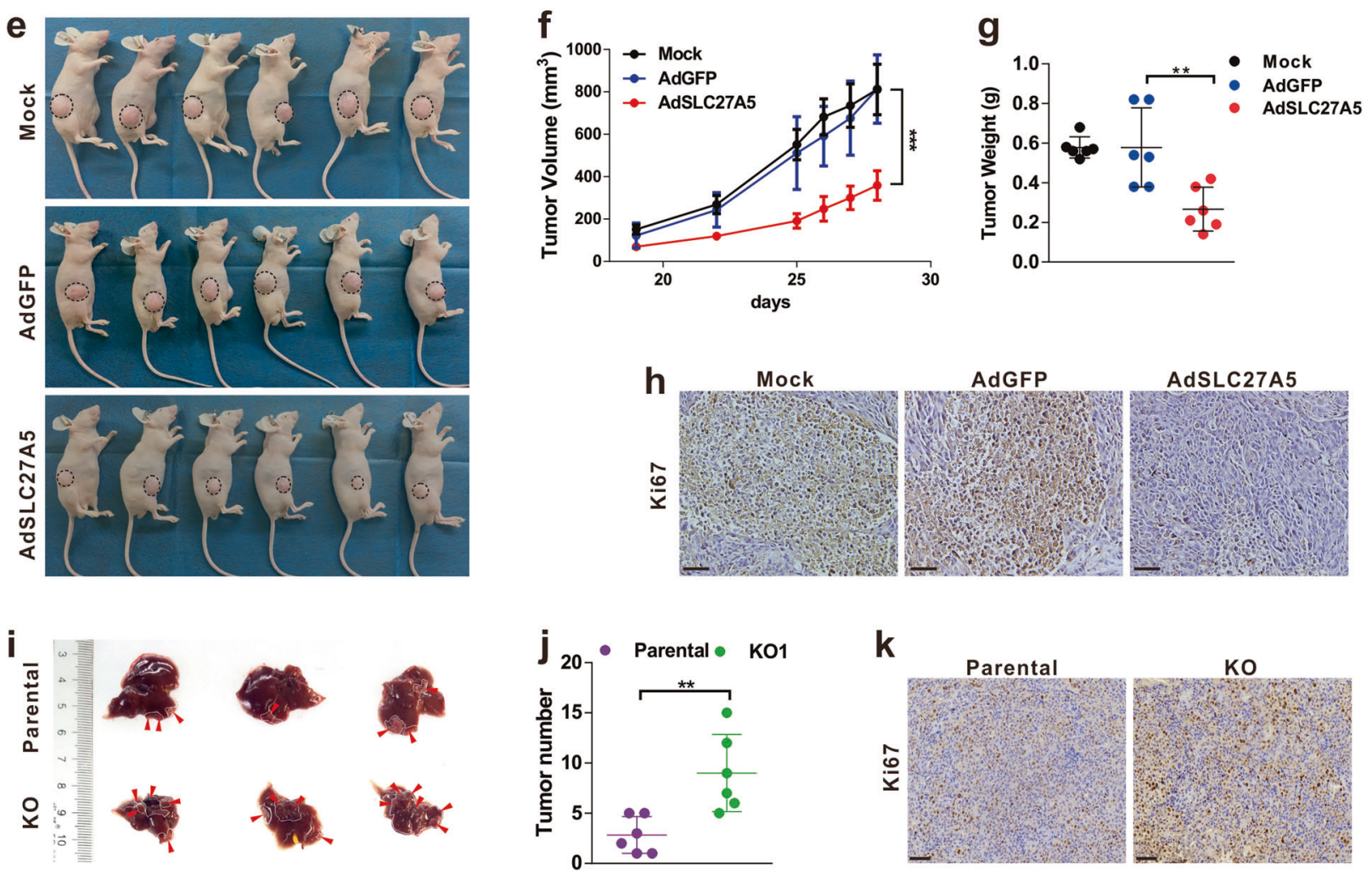

for multiple comparisons. Student's $t$-test was used to compare two groups. Pearson correlation was used to analyze the relationship between TXNRD1 expression and
SLC27A5 expression in HCC tissues. Correlations between SLC27A5 mRNA expression and individual clinicopathologic parameters were evaluated using a 
Fig. 2 SLC27A5 suppresses hepatoma cell proliferation in vitro and in vivo. Ectopic expression of SLC27A5 inhibits cell proliferation (a) and colony formation (b) in PLC/PRF/5 and SK-Hep1 cells $(n=3)$. SLC27A5 knockout promotes cell proliferation (c) and colony formation $(\mathbf{d})(n=3)$. MHCC-97H cells were infected with AdSLC27A5 or AdGFP for $36 \mathrm{~h}$. Cells $\left(5 \times 10^{6}\right.$ per site) were subcutaneously injected into the flanks of nude mice ( $n=6$ per group). e Representative tumor-bearing mice of the subcutaneous tumor model treated as indicated. f, g Graphs (mean $\pm \mathrm{SD}$ ) showing tumor growth and tumor weight. $n=6$. h Ki67 staining in tumor tissues, bar $=50 \mu \mathrm{m}$. i Orthotopic liver implantations established from SLC27A5-KO HepG2 cells. Representative liver tumors derived from SLC27A5-KO or parental HepG2 cells. j Graphs (mean \pm SD) showing liver tumor nodules, $n=6$. $\mathbf{k}$ Ki67 staining of liver tumor tissues, bar $=50 \mu \mathrm{m}$. Data are means \pm SDs. $P$ values were derived from repeated-measures ANOVA in a, c, and f; one-way ANOVA test in $\mathbf{b}, \mathbf{c}, \mathbf{d}$, and $\mathbf{g}$; unpaired, two-tailed Student's $t$-test in $\mathbf{j} ; \quad(* P<0.05, * * P<0.01$, $* * * P<0.001)$

nonparametric $\chi^{2}$ test. Probability values $(p)<0.05$ were considered statistically significant.

\section{Results}

\section{SLC27A5 expression is downregulated in HCC}

To evaluate the role of SLC27A5 (also termed as FATP5) in the carcinogenesis of HCC, we first analyzed SLC27A5 expression level in an independent cohort of $373 \mathrm{HCC}$ samples (including 50 paired tumor and normal liver tissues) from the TCGA Liver Hepatocellular Carcinoma (LIHC) dataset. SLC27A5 mRNA expression was significantly downregulated in $69.2 \%$ of clinical HCC tumors compared with normal liver tissues (Fig. 1a). Moreover, the lower SLC27A5 expression was associated with advanced tumor stage (stage I vs. stage II, $P=0.0015$; stage I vs. stage III, $P<0.001$ ) (Fig. 1b). Then, we examined SLC27A5 expression in 40 paired HCC and nontumor samples by RT-qPCR, western blotting, and immunohistochemistry (IHC) assays. The mRNA and protein expression levels of SLC27A5 were significantly lower in HCC tissues than in corresponding noncancerous tissues (Fig. 1c, d and Supplementary Fig. 1). To investigate the mechanisms underlying SLC27A5 deficiency in HCC, the methylation status of the SLC27A5 promoter were analyzed. We found that SLC27A5 expression in tumors was inversely correlated with the degree of SLC27A5 promoter methylation in the TCGA LIHC dataset (Fig. 1e). To further confirm the relevance of SLC27A5 promoter hypermethylation to its silencing in HCC, we performed direct bisulfite sequencing and revealed that the frequency of SLC27A5 gene methylation in $\mathrm{HCC}$ tissues was $51 \%$, significantly higher than that of corresponding noncancerous tissues (14\%, Fig. 1f, g), suggesting that promoter hypermethylation might be one of the reasons for SLC27A5 suppression. We further explore the relationship between SLC27A5 expression and HCC patients' survival. Patients were divided into high (top 25\%, $n=90$ ) or low (bottom 25\%, n=90) SLC27A5 expression groups based on the expression level of SLC27A5. Kaplan-Meier survival curves suggested that HCC patients with low SLC27A5 expression had poorer overall survival than patients with high SLC27A5 expression (median survival 931 vs. 2116 days; $* * * P<0.001$, Fig. 1h). In addition, deficient SLC27A5 expression was significantly associated with multiple aggressive clinicopathological characteristics of HCC, including large tumor size, poor tumor differentiation and prognosis (Table 1). Taken together, these results suggested that epigenetic silencing is a mechanism causing SLC27A5 deficiency in HCC and downregulation of SLC27A5 may contribute to disease progression and predicts poor prognosis of HCC.

\section{SLC27A5 suppresses HCC cell proliferation both in vitro and in vivo}

To evaluate the role of SLC27A5 in HCC, we first evaluated endogenous SLC27A5 levels in a collection of liver cancer cell lines and in MIHA cells. SLC27A5 expression level was substantially lower in most liver cancer cell lines, except for HepG2 cells, than in MIHA cells (Supplementary Fig. 2a). We then constructed a recombinant adenovirus encoding SLC27A5 (AdSLC27A5), and AdGFP was used as control. SLC27A5 overexpression in hepatoma cells was confirmed by the western blot analysis (Supplementary Fig. 2b). Furthermore, we generated SLC27A5-KO HepG2 cells (KO1 and KO2) by sgRNAs (Supplementary Fig. 2c) using the CRISPR/Cas9 system. The efficiency of knockout was confirmed by western blot analysis (Supplementary Fig. 2d). Overexpression of SLC27A5 significantly repressed the proliferation of both PLC/PRF/5 and SK-Hep1 cells as shown by MTS, colony formation assays and EdU incorporation (Fig. 2a, b and Supplementary Fig. 3a). KO of SLC27A5 promoted HepG2 cell proliferation (Fig. 2c, d and Supplementary Fig. 3b). Together, these results suggested that SLC27A5 represses HCC cell proliferation in vitro. To further confirm the tumor-suppressor roles of SLC27A5 in vivo, we performed xenograft tumor experiments in BALB/c nude mice. Consistent with the observations in vitro, SLC27A5 overexpression strikingly suppressed tumor growth rates compared with mock and GFP expression controls (Fig. 2e-g), which was confirmed by IHC staining of proliferation marker Ki67 (Fig. 2h). Furthermore, in orthotopic liver implantations model, liver tumors derived from SLC27A5-KO HepG2 cells displayed increased cell proliferation as indicated by larger tumor size 

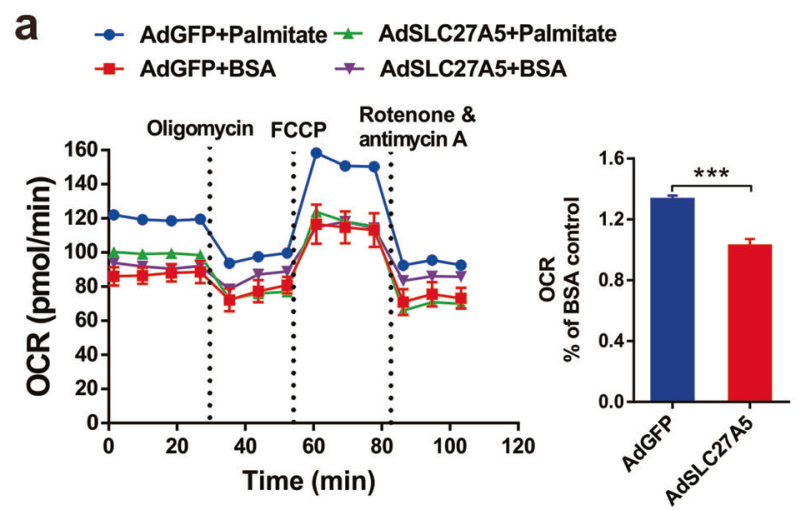

C

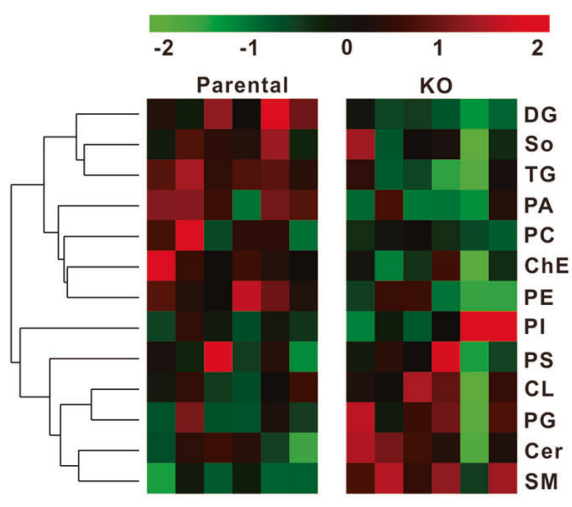

e

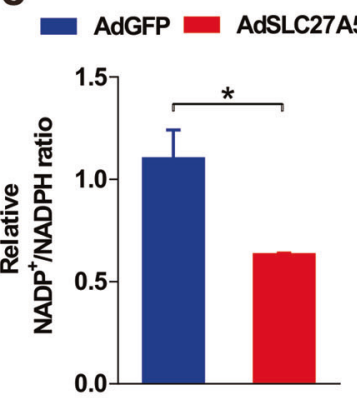

i

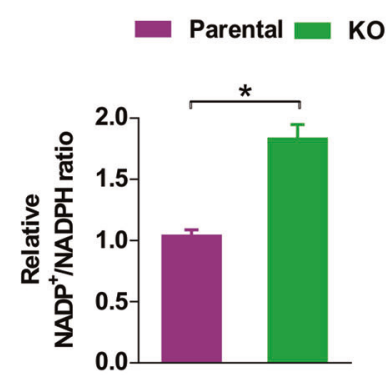

$\mathbf{f}$

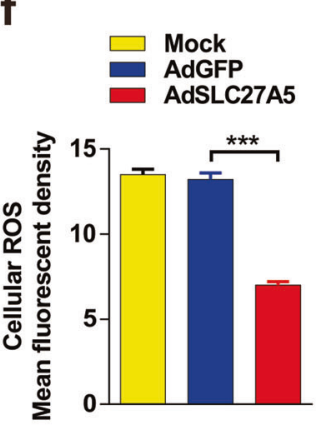

j
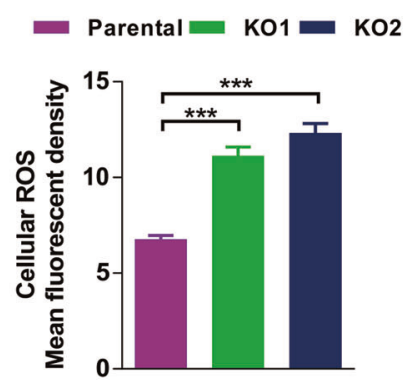

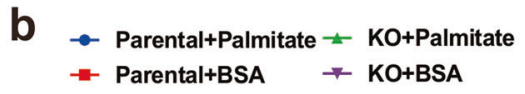

b $\rightarrow$ Parental+Palmitate $\div$ KO+Palmitate

- Parental+BSA $*$ KO+BSA
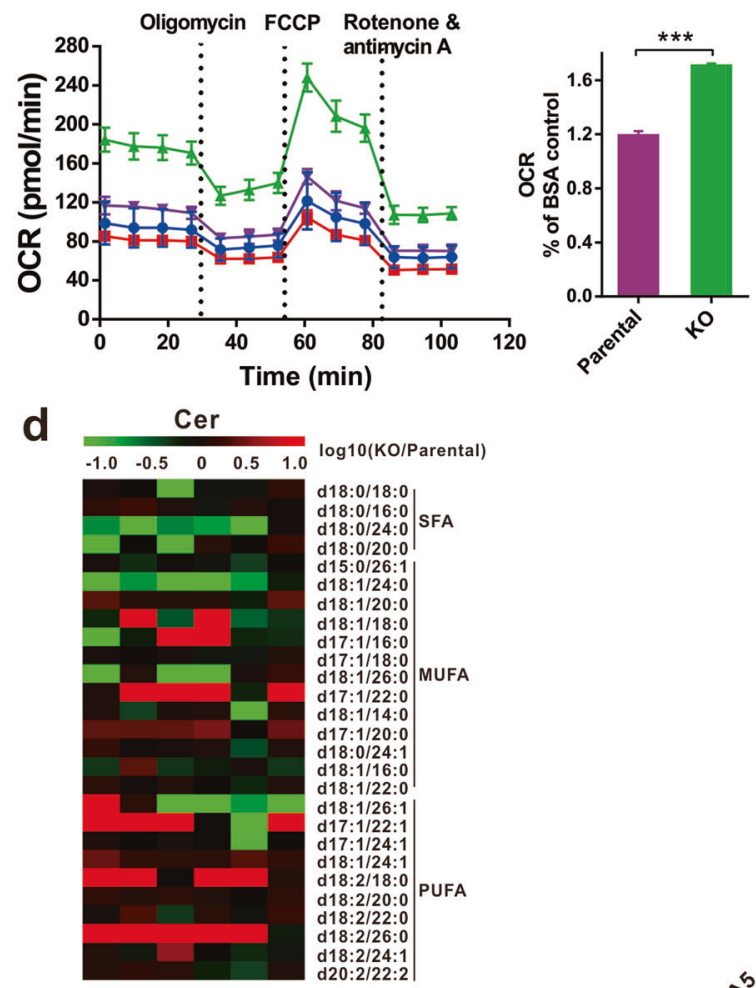

$\log 10($ KO/Parental)

d18:0/18:0 d18:0/16:0 SFA d18:0/20:0 d15:0/26:1 d18:1/24:0 d18:1/20:0 d18:1/18:0 d17:1/16:0 d18:1/26:0 MUFA d17:1/22:0 d18:1/14:0 d17:1/20:0 d18:0/24:1 d18:1/16:0 d18:1/26:1 d17:1/24:1 d18:1/24:1 d18:2/18:0 PUFA d18:2/20:0 d18:2/22:0 d18:2/2:1 d20:2/22:2
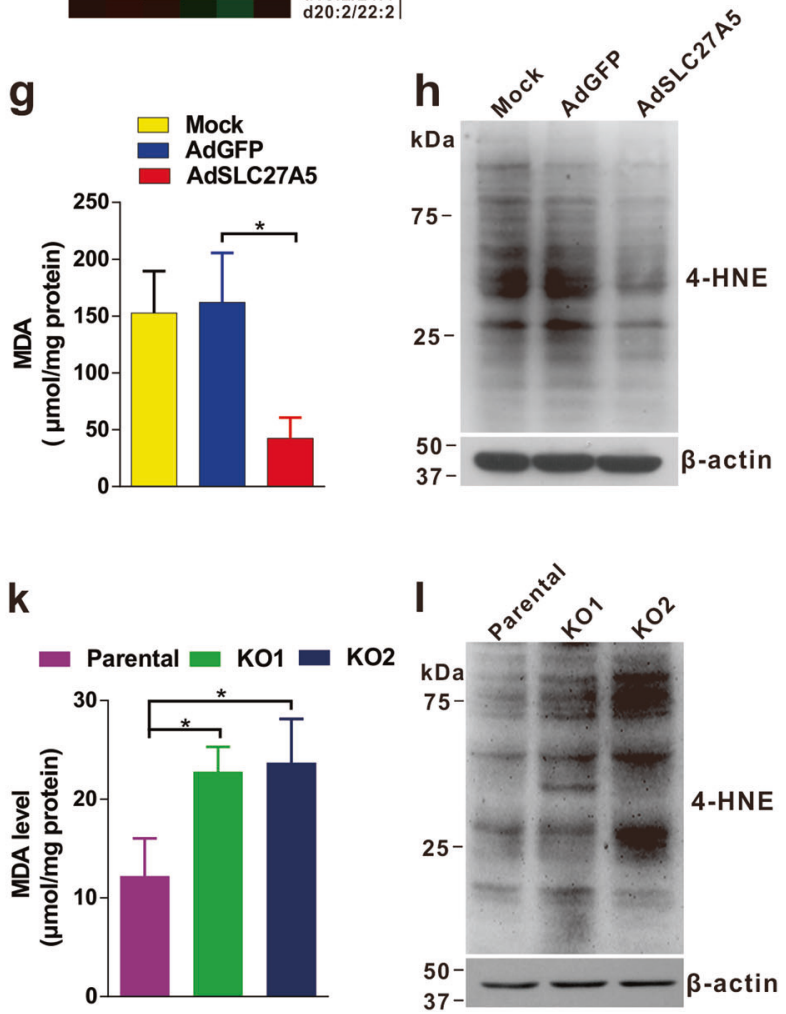

in vitro and in vivo gain- and loss-of-function studies clearly demonstrated that SLC27A5 functions as a tumor suppressor.
(Fig. 2i), increased number of tumor nodules (Fig. 2j), and enhanced Ki67 staining (Fig. 2k) compared with parental HepG2 cells-derived liver tumors. Taken together, these 
Fig. 3 Loss of SLC27A5 enriches polyunsaturated lipids and elevates oxidative stress and lipid peroxidation. FAO levels in SLC27A5-OE PLC/PRF/5 (a) and SLC27A5-knockout HepG2 cells (b) after nutrient starvation. Increases in maximal respiration beyond the BSA control indicate utilization of exogenous FAs $(n=3)$. Heatmap of lipid abundances (c) and ceramide (d) in SLC27A5-KO and parental HepG2 cells $(n=6)$. DG diacylglyceride, So sphingosine, TG triacylglyceride, PA phosphatidic acids, PC phosphatidylcholine, ChE cholesteryl esters, PE phosphatidylethanolamine, PI phosphatidylinositol, PS phosphatidylserine, CL cardiolipins, PG phosphatidylglycerols, Cer ceramides, SM sphingomyelin, SFA saturated fatty acid, MUFA monounsaturated fatty acid, PUFA polyunsaturated fatty acid. e-h Levels of $\mathrm{NADP}^{+} / \mathrm{NADPH}, \mathrm{ROS}$, and lipid peroxidation in SLC27A5-OE cells. e NADP ${ }^{+}$/NADPH ratio in SLC27A5-OE cells. f Intracellular ROS were measured by CellROX Orange fluorogenic probe. Scale bar: $10 \mu \mathrm{M}$. Mean fluorescence intensity was analyzed with Image Pro Plus software. g MDA levels in SLC27A5-OE cells. MDA concentrations were normalized to total protein content $(n=3$, performed in triplicate). $\mathbf{h}$ 4-HNE-induced protein modification was examined by western blot analysis. Levels of NADP ${ }^{+} / \mathrm{NADPH}$ ratio (i), ROS (j), and lipid peroxidation, including MDA levels (k) and 4HNE-induced protein modification (l), in SLC27A5-knockout HepG2 cells $(n=3)$. Data are presented as means \pm SDs. $P$ values were derived from an unpaired, two-tailed Student's $t$-test in $\mathbf{a}, \mathbf{b}, \mathbf{e}$, and $\mathbf{i}$; one-way ANOVA test were performed in $\mathbf{f}, \mathbf{g}, \mathbf{j}$ and $\mathbf{k}\left({ }^{*} P<0.05\right.$, $* * * P<0.001)$

\section{Loss of SLC27A5 elevates polyunsaturated lipids and ROS level}

As SLC27A5 plays an important role in lipid metabolism, we then tested whether SLC27A5 affects cellular metabolism of hepatoma cells using the Seahorse Bioscience $\mathrm{XFe} 24$ analyzer. FAs are catabolized by $\mathrm{FAO}$, thus providing an alternative energy source for tumor cells. The results indicate that FAO was significantly decreased in PLC/PRF/5 cells overexpressing SLC27A5 (Fig. 3a) and significantly increased in SLC27A5-KO versus parental HepG2 cells during metabolic stress (Fig. 3b). We then explored the lipidomic profiles in SLC27A5-deficient hepatoma cells. We detected significantly higher amounts of total ceramide (Cer) and sphingomyelin (SM), whereas decreased amounts of total sphingosine (So) in SLC27A5KO HepG2 cells (Fig. 3c), indicating disrupted lipid composition in the sphingolipid metabolism pathway. Moreover, polyunsaturated fatty acids (PUFA)-containing lipids such as PUFA-Cer, PUFA-phosphatidylcholine (PC) and PUFA-phosphatidylethanolamine (PE) were found markedly increased (Fig. 3d and Supplementary Fig. 4). Considering that the mitochondrial respiratory chain is a major source of ROS [22], and the accumulation of PUFA lipids would be expected to account for an increase in ROS production as well [23], we measured $\mathrm{NADP}^{+} / \mathrm{NADPH}$ ratios, intracellular ROS, and lipid peroxidation levels in hepatoma cells. As expected, overexpression of SLC27A5 decreased the intracellular $\mathrm{NADP}^{+} / \mathrm{NADPH}$ ratio (Fig. 3e), and reduced intracellular
ROS (Fig. 3f and Supplementary Fig. 5a) and lipid peroxidation, including the malondialdehyde (MDA) level (Fig. 3g) and 4-HNE-induced protein modification (Fig. 3h). Loss of SLC27A5 had inverse effects on ROS production and lipid peroxidation in $\mathrm{HepG} 2$ cells, including an increased intracellular $\mathrm{NADP}^{+} / \mathrm{NADPH}$ ratio (Fig. 3i), and elevated intracellular ROS (Fig. 3j and Supplementary Fig. 5b) and MDA levels (Fig. 3k), and 4HNE-induced protein modification (Fig. 31). Furthermore, ROS generation and MDA levels (Supplementary Fig. 5c-d) were reduced in SLC27A5-overexpressing tumor mass, whereas elevated oxidative stress, including increased intracellular ROS and MDA levels (Supplementary Fig. 5e-f), were found in liver tumors derived from SLC27A5-deficient hepatoma cells. Based on these observations, we assumed that loss of SLC27A5 elevates PUFA lipids, induces ROS production and lipid peroxidation.

\section{4-HNE modification of KEAP1 and activation of NRF2 signaling in SLC27A5 deficient hepatoma cells}

The KEAP1/NRF2 pathway plays a vital role in the protection of cells against the cumulative damages of oxidants/ electrophiles [24]. A variety of electrophilic and oxidants, such as $15 \mathrm{~d}_{-}-\mathrm{PGJ}_{2}$, tBHQ, 4-HNE, and $\mathrm{H}_{2} \mathrm{O}_{2}$, have been reported to react with cysteine thiols of KEAP1 and potentiate NRF2 activity [25]. Thus, we examined whether SLC27A5 could regulate 4-HNE-induced modification of KEAP1 in hepatoma cells. Strikingly, modification of KEAP1 by 4-HNE was decreased in PLC/PRF/5 cells overexpressing SLC27A5, whereas 4-HNE modification of KEAP1 was increased upon loss of SLC27A5 (Fig. 4a, b), suggesting that SLC27A5 indeed represses modification of KEAP1 by 4-HNE.

To identify the major cysteine sensors involved in the response to 4-HNE exposure, we transfected SLC27A5KO HepG2 cells with Flag-tagged KEAP1. Mass spectrometry of Flag-immunoprecipitated KEAP1 identified Cys513 and Cys518 as the major residues of KEAP1 involved in sensing 4-HNE in vitro (Fig. 4c). As one of the most readily modified cysteines in KEAP1 [26], substitution of Cys151 with serine do not respond to a large group of electrophiles such as 4-HNE, leading to continuous degradation of NRF2. In contrast, substitution of Cys151 with alanine leads to the accumulation of NRF2 [27], which was reproduced in our experiment (Fig. 4d, e). We then generated PLC/PRF/5 cells expressing C513A, C513S, C518A, or C518S KEAP1 mutants and found that NRF2 protein expression were dramatically reduced in PLC/PRF/5 cells expressing KEAP1 C513A/S and C518A/S mutants compared with WT-KEAP1-transduced cells upon 4-HNE stimulation, suggesting that these 

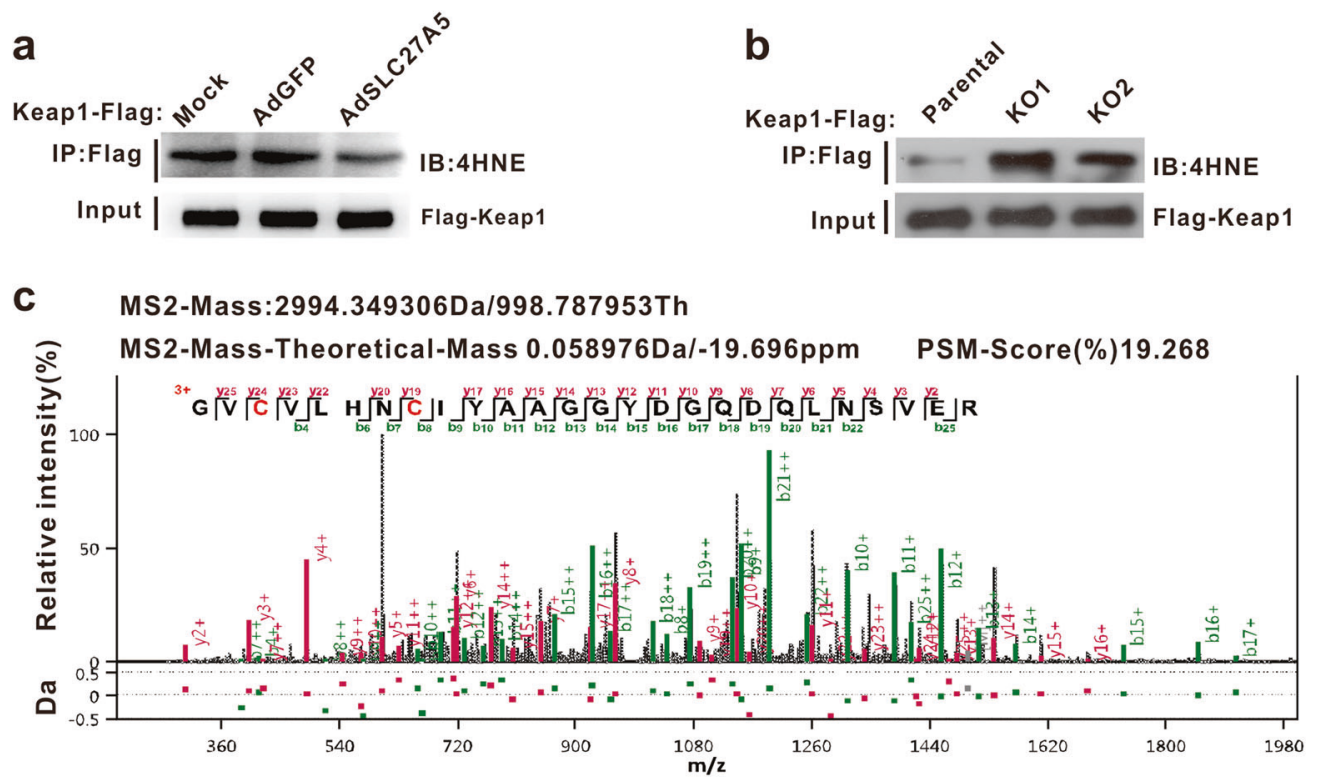

MS2-Mass:2994.349375Da/998.787976Th
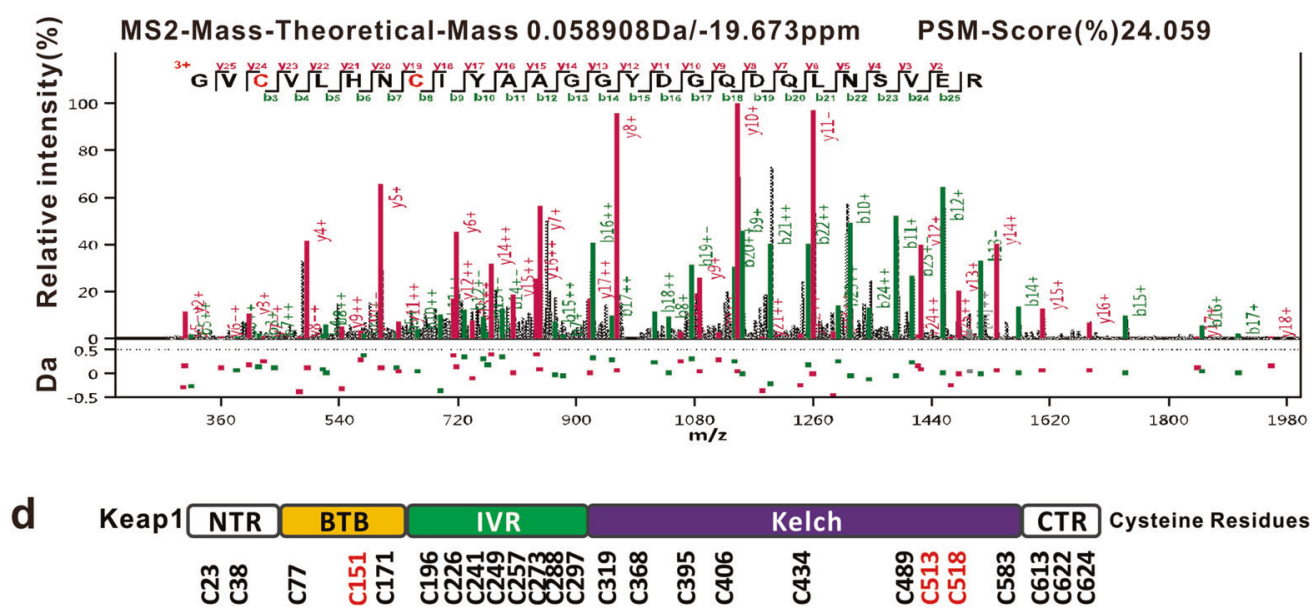

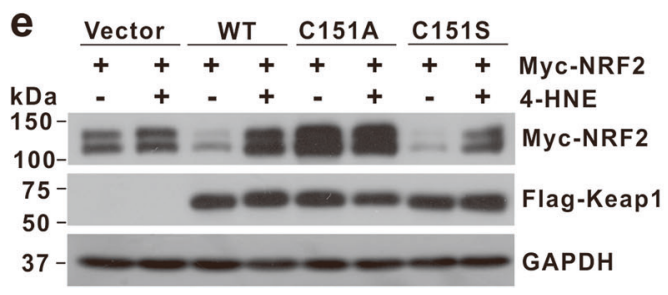

Fig. 4 SLC27A5 represses oxidative modification of KEAP1 by $4-$ HNE in hepatoma cells. Immunoprecipitation of 4-HNE-modified KEAP1. SLC27A5-overexpressing PLC/PRF/5 (a) and SLC27A5knockout HepG2 cells (b) were infected with adenoviruses expressing Flag-KEAP1 for $48 \mathrm{~h}$, followed by Flag immunoprecipitation and western blot analysis of 4-HNE. c 4-HNE alkylation of human KEAP1 cysteine residues Cys513 and Cys518 was identified in SLC27A5knockout HepG2 cells by MS/MS analysis. d Schematic diagram

KEAP1 mutants could not effectively respond to 4 -HNE modification and thus alleviate NRF2/ARE activation (Fig. 4f). These results suggested that both Cys513 and Cys518 are important sensors for 4-HNE-mediated activation of NRF2 signaling.

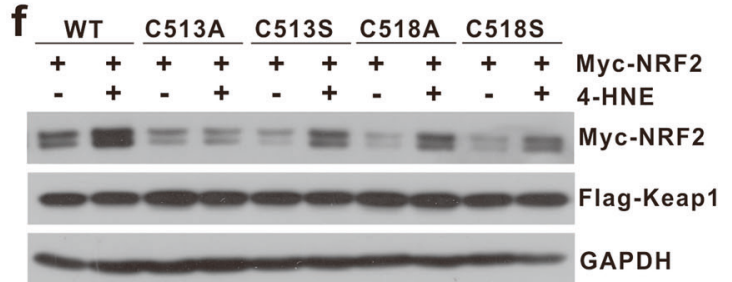

showing the NTR, BTB, IVR, Kelch, and CTR domains of KEAP1. The cysteine residues as well as KEAP1 mutants with substitution of a cysteine (C) to alanine (A) or cysteine (C) to serine (S) are indicated. e, f Constructs expressing Flag-KEAP1 (WT), or Flag-KEAP1 C151A, C151S, C513A, C513S, C518A, or C518S mutant were cotransfected into PLC/PRF/5 cells with plasmids expressing Myc-Nrf2. At $48 \mathrm{~h}$ after transfection, cells were treated with 4-HNE $(20 \mu \mathrm{M}, 2 \mathrm{~h})$ before blotting for Myc-Nrf2

\section{Loss of SLC27A5 activates the KEAP1/NRF2 pathway in HCC cell lines}

To further confirm the relationship between the SLC27A5 and KEAP1/NRF2 pathway, transcriptional activities of 
a

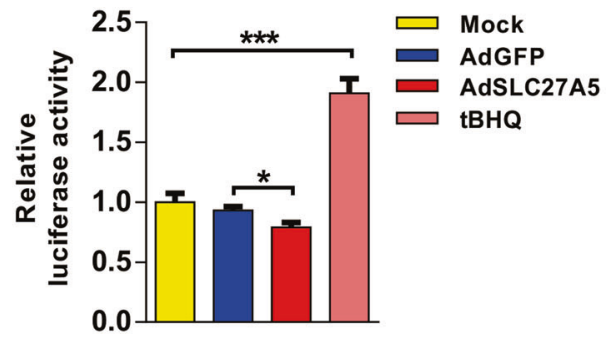

C
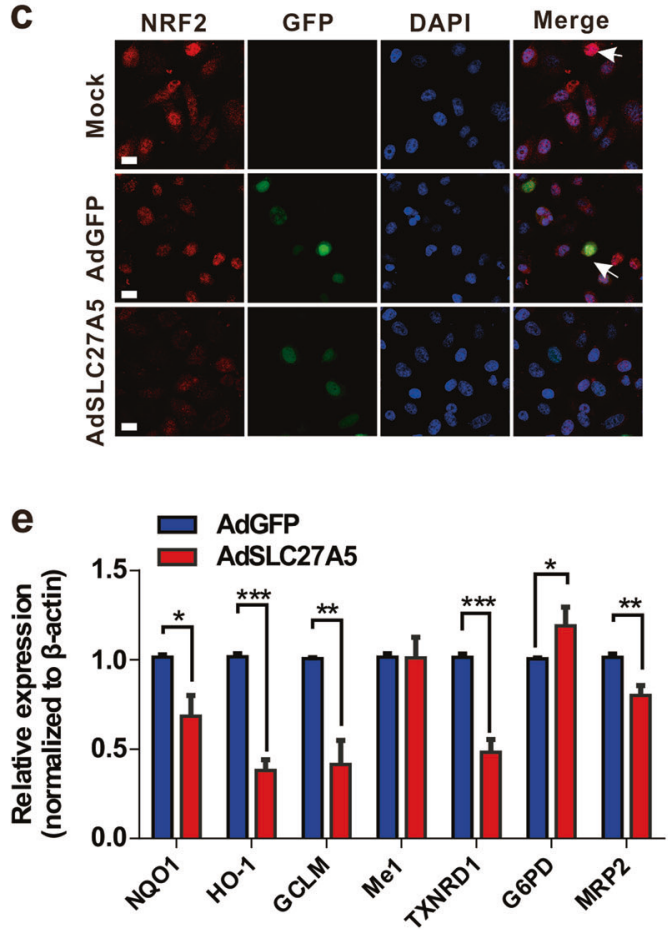

g

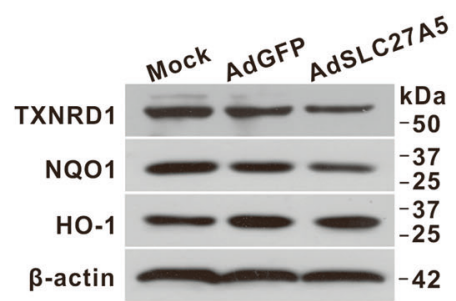

h

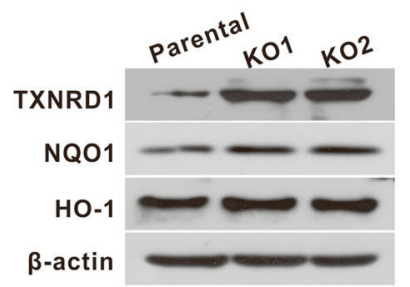

NRF2 modulated by SLC27A5 were determined by ARE reporter assays. SLC27A5 overexpression decreased AREdriven luciferase activity to $76.8 \%$ (Fig. 5a), whereas AREluciferase activity was increased by $\sim 1.7$-fold when SLC27A5 was knocked out (Fig. 5b). Meanwhile,
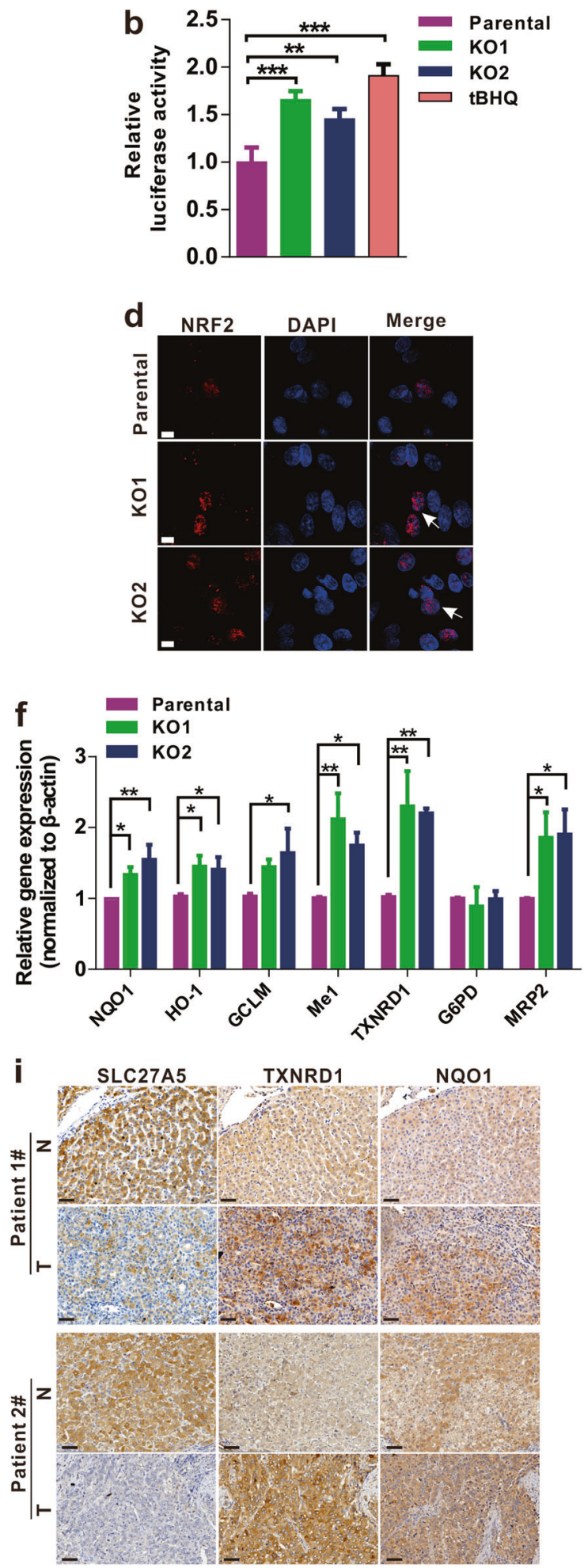

overexpression of SLC27A5 decreased nuclear translocation of NRF2 (Fig. 5c and Supplementary Fig. 6a), whereas KO of SLC27A5 had an opposite effect on NRF2 distribution in hepatoma cell lines (Fig. 5d and Supplementary Fig. 6b). We further evaluated the mRNA and protein levels 
Fig. 5 SLC27A5 inhibits NRF2 antioxidative pathway. a, b NRF2mediated gene transcriptional activities in SLC27A5-OE or -KO HCC cells as assessed by ARE-luciferase assays $(n=3)$. Tertbutylhydroquinone (tBHQ, $100 \mu \mathrm{M})$ was used as a positive control. c Intracellular localization of NRF2 (red) in SLC27A5-OE PLC/PRF/5 cells by the immunofluorescence assay. Nuclei are stained blue with DAPI. Bar $=50 \mu \mathrm{m}$. d Intracellular localization of NRF2 (red) in SLC27A5-KO HepG2 cells. Nuclei are stained blue with DAPI. Bar = $10 \mu \mathrm{m}$. e, f mRNA expression of NRF2-downstream target genes NQO1, HO-1, GCLM, Me1, TXNRD1, G6PD, and MRP2 in SLC27A5-OE PLC/PRF/5 (e) and SLC27A5-KO HepG2 cells (f). $(n=3$, performed in triplicate). Protein expression of NRF2downstream target genes NQO1, HO-1, and TXNRD1 in SLC27A5OE PLC/PRF/5 (g) and SLC27A5-KO HepG2 cells (h). i Representative IHC staining of SLC27A5, TXNRD1, and NQO1 in serial tissue slices. HCC tissues (T) and adjacent nontumor tissues (N) $($ bar $=50 \mu \mathrm{m})$. Data are means \pm SDs. $P$ values were derived from oneway ANOVA test in $\mathbf{a}, \mathbf{b}$, and $\mathbf{f}$; an unpaired, two-tailed Student's $t$-test in $\mathbf{e}(* P<0.05, * * P<0.01, * * * P<0.001)$

of representative NRF2-downstream target genes. The mRNA levels of NQO1, HO-1, GCLM, TXNRD1, and $M R P 2$ were significantly decreased in PLC/PRF/5 cells overexpressing SLC27A5 (Fig. 5e), but increased by 1.5- to 2.0-fold in SLC27A5-KO cells (Fig. 5f). Meanwhile, the protein levels of TXNRD1 and NQO1 were found significantly decreased in SLC27A5-overexpressing cells (Fig. 5g), while increased in SLC27A5-KO cells (Fig. 5h). The IHC assays also confirmed the negative correlation between SLC27A5 and TXNRD1 or NQO1 expression (Fig. 5i). Based on these results, we assumed that the loss of SLC27A5 activates the KEAP1/NRF2 pathway and upregulates downstream genes expression by elevated ROS production and lipid peroxidation.

\section{SLC27A5 expression negatively correlates with TXNRD1 expression}

The TrxR/Trx system is one of the central players in the cellular redox regulation network and TXNRD1 is an important target gene of the KEAP1/NRF2 pathway, thus we analyzed the expression patterns between SLC27A5 and TXNRD1. Both mRNA and protein levels of TXNRD1 were significantly downregulated upon SLC27A5 overexpression (Fig. 6a, b). In contrast, TXNRD1 mRNA and protein levels were increased in SLC27A5-KO cells as compared with parental cells (Fig. 6c, d). Furthermore, we used mouse embryonic fibroblasts (MEFs) derived from Nrf2 $2^{-1-}$ mice to investigate whether SLC27A5-mediated TXNRD1 inhibition indeed depends on the NRF2 pathway. Interestingly, SLC27A5-induced TXNRD1 repression in Nrf2 ${ }^{+/+}$MEFs was not observed in Nrf2 ${ }^{-/-}$MEFs (Fig. 6e), suggesting that SLC27A5 decreases TXNRD1 expression through the NRF2 pathway. In addition, analysis of the 40 paired HCC and nontumor tissues confirmed that TXNRD1
mRNA expression was significantly upregulated in HCC (Fig. 6f). We observed a negative correlation between TXNRD1 and SLC27A5 expression $(r=-0.35, P=0.02$, Fig. $6 \mathrm{~g}$ ) in 20 paired HCC tissues. The association between increased TXNRD1 and decreased SLC27A5 expression was corroborated by the results of immunoblot and IHC assays of human HCC tissues (Fig. 6h, i) and tumor tissues derived from tumor-bearing mice (Supplementary Fig. 7). Taken together, the data indicated that decreased SLC27A5 expression is correlated with increased TXNRD1 expression in HCC cells and tissues.

\section{Blockade of NRF2/TXNRD1 sensitizes HCC cells to sorafenib treatment}

Constitutive activation of NRF2 has been found to be related to chemoresistance and poor patient outcome [28], which led us to hypothesize that blockade of NRF2 may sensitize HCC cells to sorafenib treatment. Indeed, our study revealed that NRF2 blockade with brusatol significantly sensitizes SLC27A5-deficient hepatoma cells to sorafenib treatment (Supplementary Fig. 8a, b). We further examined whether targeting TXNRD1 could also improve the response of hepatoma cells to sorafenib. Importantly, knockdown of TXNRD1 enhanced sorafenib-induced growth inhibition in SLC27A5-KO HepG2 cells (Fig. 7a, b). The synergistic lethal effects of sorafenib and TXNRD1 inhibition were confirmed in the orthotopic implantation model (Fig. 7c-e). Moreover, enhanced apoptosis and excess ROS accumulation were also observed in the orthotopic implantation model (Fig. 7f, g). Consistent with the findings in SLC27A5-KO HepG2 cells, TXNRD1 silencing (shTXNRD1) or the TXNRD1 inhibitor auranofin could also improve the response of $\mathrm{MHCC}-97 \mathrm{H}$ cells to sorafenib in vitro (Supplementary Fig. 8c-f) and in vivo (Supplementary Fig. 9).

Collectively, these data suggested that loss of SLC27A5 elevates ROS and lipid peroxidation due to an increase in polyunsaturated lipids, thus leading to KEAP1/NRF2 pathway activation and TXNRD1 upregulation. Sorafenib and NRF2/TXNRD1 inhibitors exerts synergistic antitumor effects in HCC (Fig. 7h).

\section{Discussion}

Our data together describe a SLC27A5/NRF2/ TXNRD1 signaling axis that regulates tumorigenicity and responses to sorafenib treatment in HCC. SLC27A5 deficiency in HCC is associated with its promoter hypermethylation and may predict poor prognosis and advanced disease progression. Constitutively SLC27A5 depletion increases intracellular PUFA lipids and ROS level, thus activating the KEAP1/NRF2 pathway, enhancing TXNRD1 


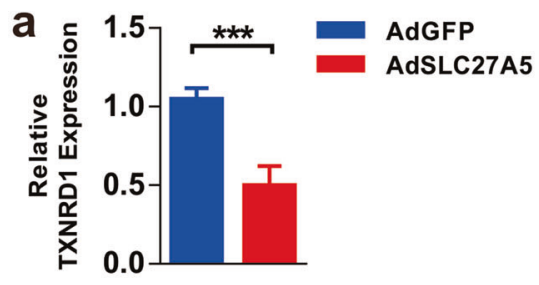

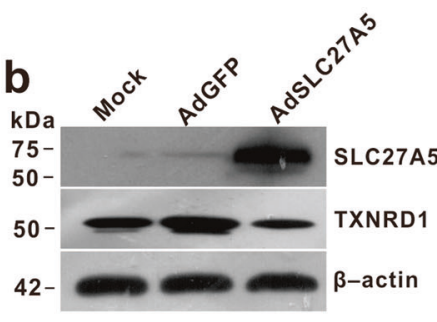

PLC/PRF/5

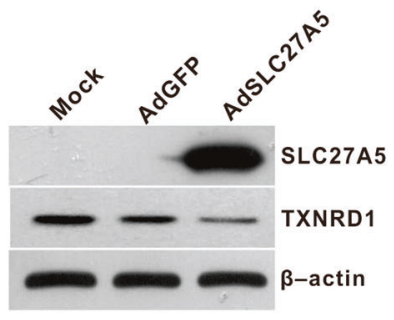

SK-Hep1
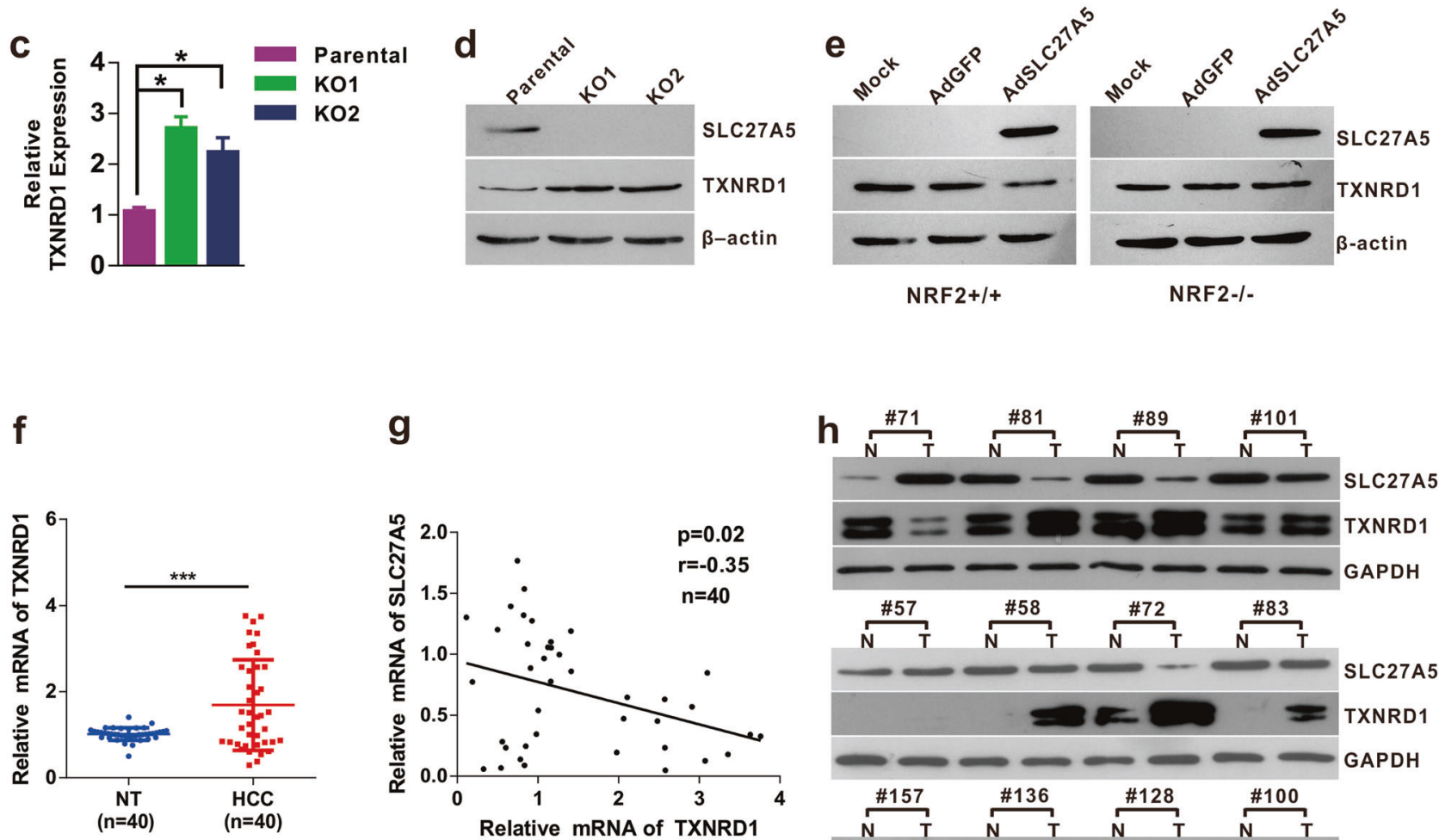

h
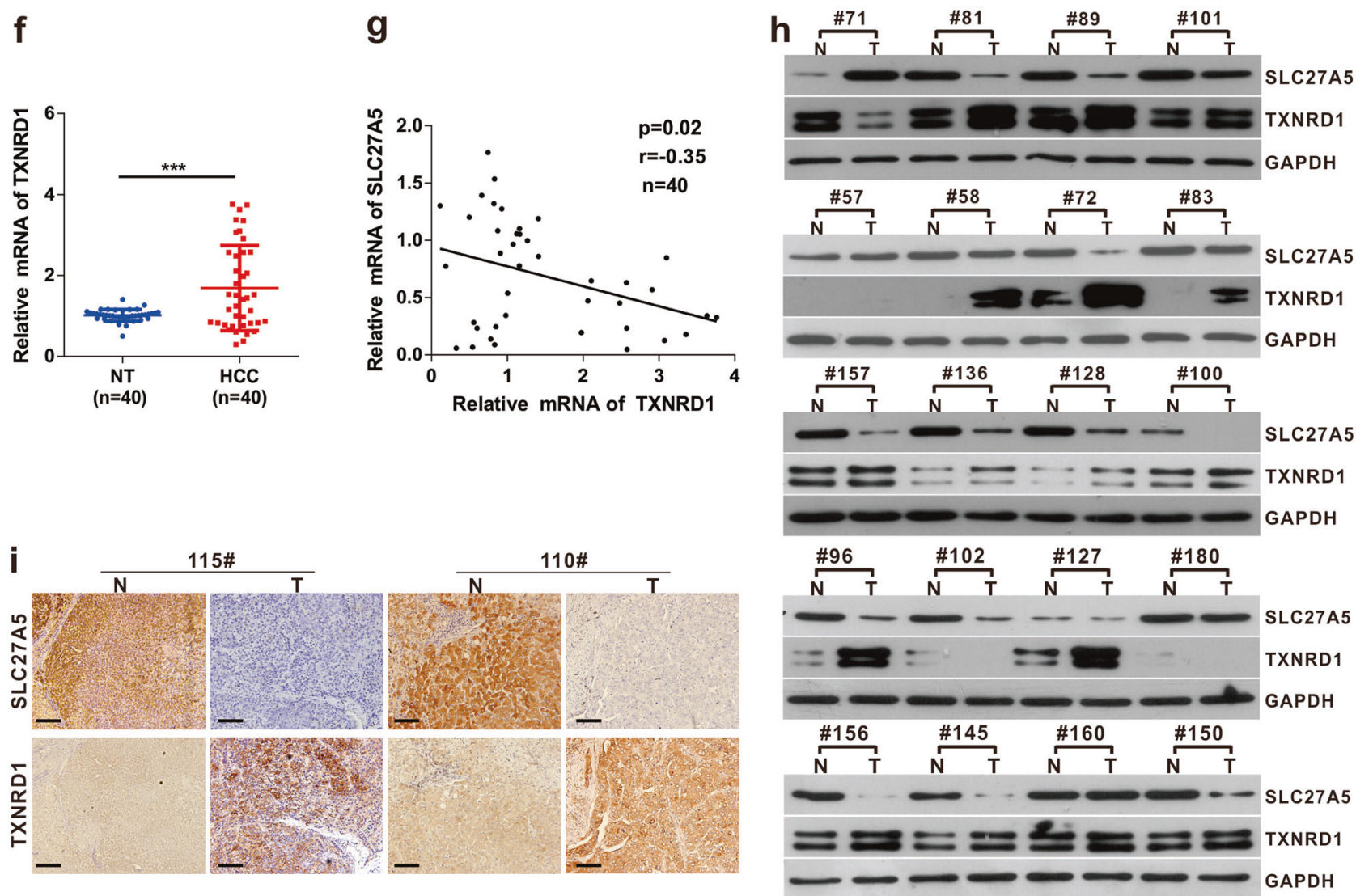

expression, and promoting hepatoma cell growth. Genetic or pharmacologic inhibition of NRF2/TXNRD1 markedly enhances the antitumor activity of sorafenib (Fig. 7h).

Recently, some molecules involved in FA metabolism has been proved to regulate tumor growth and metastasis.
Four FA transporters, including CD36, plasma membrane FA-binding protein, SLC27A2, and SLC27A5, are known to be responsible for FA uptake in the liver [29]. Upregulation of CD36 has recently been reported to enhance FA uptake and promote epithelial-mesenchymal transition in 
Fig. 6 SLC27A5 negatively correlates with TXNRD1 expression in hepatoma cells and HCC tissues. mRNA and protein expression of TXNRD1 in SLC27A5-overexpressing cells $(\mathbf{a}, \mathbf{b})$ and SLC27A5-KO cells $(\mathbf{c}, \mathbf{d})$. Data represent the means $\pm \mathrm{SD}$. $(n=3$, performed in triplicate). ${ }^{*} P<0.05, * * * P<0.001$. e Immunoblot analysis of TXNRD1 in mouse embryonic fibroblasts (MEFs) lysates from $\mathrm{Nrf}^{+/+}$and $N r f 2^{-1-}$ cells infected with AdSLC27A5 or AdGFP. $\beta$-actin was used as an internal control. $f$ The mRNA expression of SLC27A5 and TXNRD1 in 40 pairs of HCC tissues and adjacent nontumorous tissues $(\mathrm{HCC} / \mathrm{NT})$. Data are means $\pm \mathrm{SD}$. $* * * P<0.001$, Wilcoxon's signedrank tests were used. g Correlation analysis of SLC27A5 and TXNRD1 mRNA expression in 40 paired HCC and nontumor tissues $\left({ }^{*} P<0.05, r=-0.35\right.$, Pearson's correlation). $\mathbf{h}$ Immunoblot analysis of SLC27A5 and TXNRD1 protein expression in 20 paired HCC and nontumor tissues. i Representative IHC staining of SLC27A5 and TXNRD1 in serial tissue slices from 20 paired HCC tissues and adjacent nontumor tissues $(\mathrm{T} / \mathrm{N}) .(\mathrm{bar}=50 \mu \mathrm{m})$

HCC [30]. SLC27A2 increases the arachidonic acid uptake and PGE2 synthesis in PMN-MDSCs from multiple cancers and supports tumor growth and escapes immune surveillance [31]. The expression of SLC27A1 and CD36 are increased in melanoma [32, 33], while decreased in endometrial cancer tissues [34], suggesting that FA transporters may play contrasting roles in tumorigenesis in different tumor types.

Our study highlights a tumor suppressor role of SLC27A5 in HCC and first demonstrated that SLC27A5 is critical to the maintenance of redox homeostasis. ROS are important messenger molecules that regulate diverse redox signaling pathways [35]. Cancer cells usually harbor elevated ROS due to uncontrolled proliferation and high metabolic rate. In our study, we demonstrated that SLC27A5 plays an important role in combating oxidative stress both in vitro and in vivo. First, the sphingolipid metabolism pathway was differentially regulated upon SLC27A5 deficiency. The total content of So was decreased, whereas SM and Cer were increased, which may suggest the increased de novo Cer synthetic and the salvage pathway [36]. We postulate that increased Cers synthesis induced ROS accumulation in SLC27A5-deficiency cells, which is consistent with previous studies indicating that metabolism of Cer promotes the generation of ROS in neuroblastoma cells [37] and C6-Cer increases ROS generation in human endothelial cells [38]. Second, polyunsaturated lipids such as PUFA-Cer, PUFA-PC, and PUFA-PE were remarkably increased in SLC27A5deficient cells. This may also contribute to elevated ROS level, as polyunsaturated lipids can generate excess ROS via increased peroxidation and increased activity of NADPH oxidase [23, 39]. Third, the OCR upon palmitic acid stimulation in SLC27A5-KO HepG2 cells under glucose deprivation was increased, which may increase electron flux in the mitochondrial respiratory chain, resulting in elevated ROS generation.
The KEAP1/NRF2 pathway is one of the most frequently mutated pathways in HCC [40] and play key roles in restoring cellular redox balance. KEAP1, a critical negative regulator of NRF2, is a substrate adaptor protein for a cullin 3-containing E3 ubiquitin ligase. Under oxidative stress conditions, cysteine modifications cause conformational changes in the KEAP1, leading to the activation of NRF2. Our study highlights an important role of the SLC27A5 deficiency in activating KEAP1/NRF2/TXNRD1 signaling axis in HCC. In support of this notion, NRF2 nuclear translocation and its targets including TXNRD1 were dramatically increased in hepatoma cells and mice tumor xenografts with SLC27A5 KO. Mechanistically, we have demonstrated that SLC27A5 deficiency can activate the KEAP1/NRF2 pathway by elevated oxidative stress i.e., the accumulation of ROS and the subsequent lipid peroxidation products including MDA and 4-HNE. Furthermore, our data demonstrated that 4-HNE modification of KEAP1 was increased upon loss of SLC27A5, and Cys513 and Cys518 in the Kelch domain of KEAP1 were directly modified by 4HNE in SLC27A5-KO cells, thus triggering NRF2 activation and subsequent TXNRD1 expression. Previous reports have identified Cys 151 is one of the most readily modified cysteines in KEAP1 [41]. Amino-acid substitution analyses have suggested that Cys151, Cys273, and Cys288 of KEAP1 are responsible for 4-HNE sensing [42]. However, whether these cysteine residues can be directly modified by 4-HNE requires further investigation.

Antioxidant-related pathways have been recognized as attractive therapeutic targets for cancer cells. NRF2, a key regulator of antioxidant responses and detoxification, is regarded as a driver of cancer progression, metastasis and chemotherapy resistance [43]. TXNRD1, an antioxidant enzyme, is upregulated in various human malignancies and has been shown to play important roles in drug resistance. Previous studies revealed that overexpression of TXNRD1 modulates drug-specific cytotoxic responses [44] and inhibition of the TrxR enhances the efficacy of some chemotherapeutics, such as improving ibrutinib's anti-EGFR activity in lung cancer [45]. Our current study showed that targeting of NRF2/TXNRD1 is an effective approach to inhibit HCC growth. Importantly, a combination of sorafenib with NRF2/TXNRD1 inhibition has synergistic antitumor effects in SLC27A5-deficient hepatoma cells.

In conclusion, this study reveals the importance of SLC27A5 in retarding liver tumor growth via inhibiting NRF2/TXNRD1 signaling axis, providing a mechanistic link between disrupted lipid metabolism and redox homeostasis. Our study not only provides a potential prognostic marker for HCC, but also reveals a clinical opportunity involving combined NRF2/TXNRD1 inhibitors and sorafenib for treating patients with HCC. 


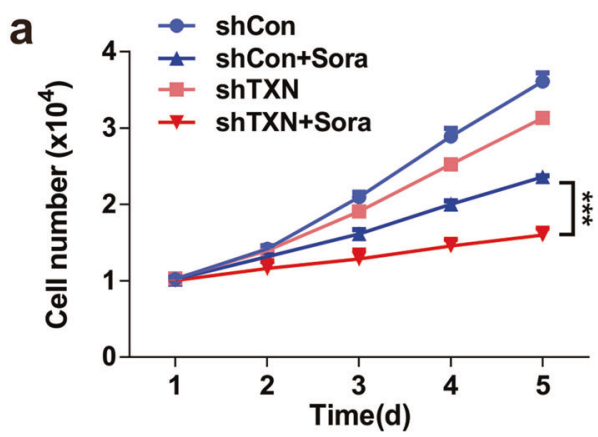

b
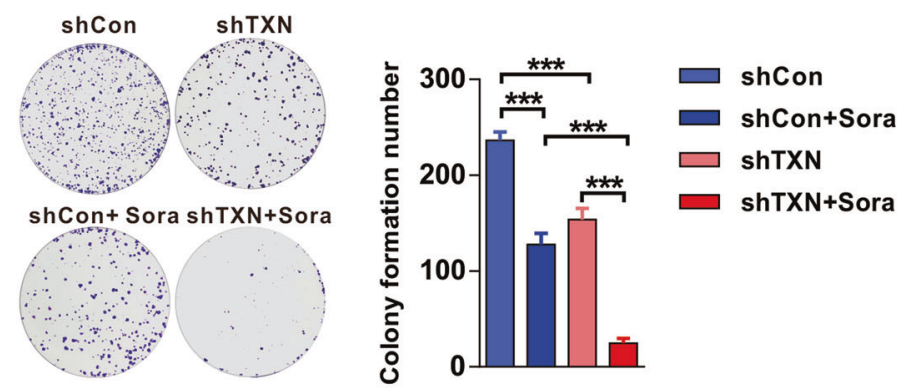

C
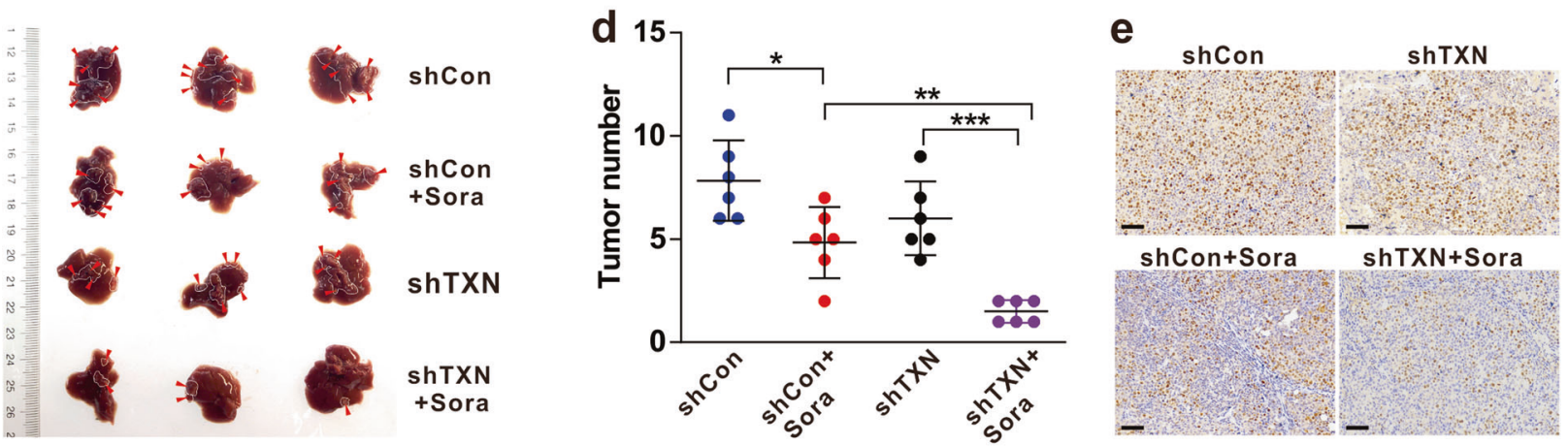

f
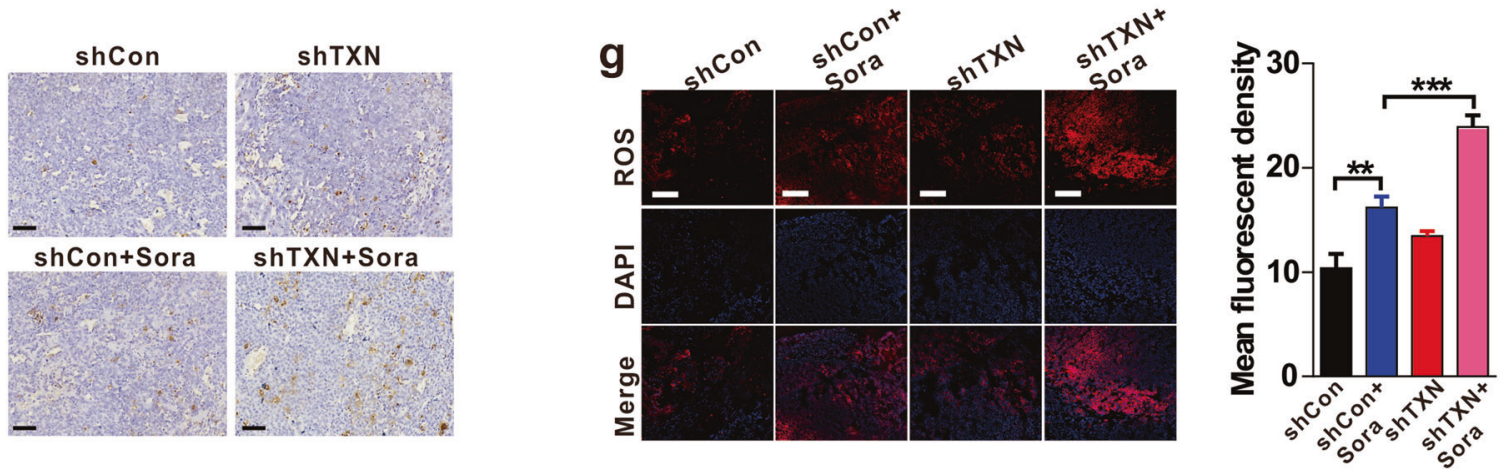

h

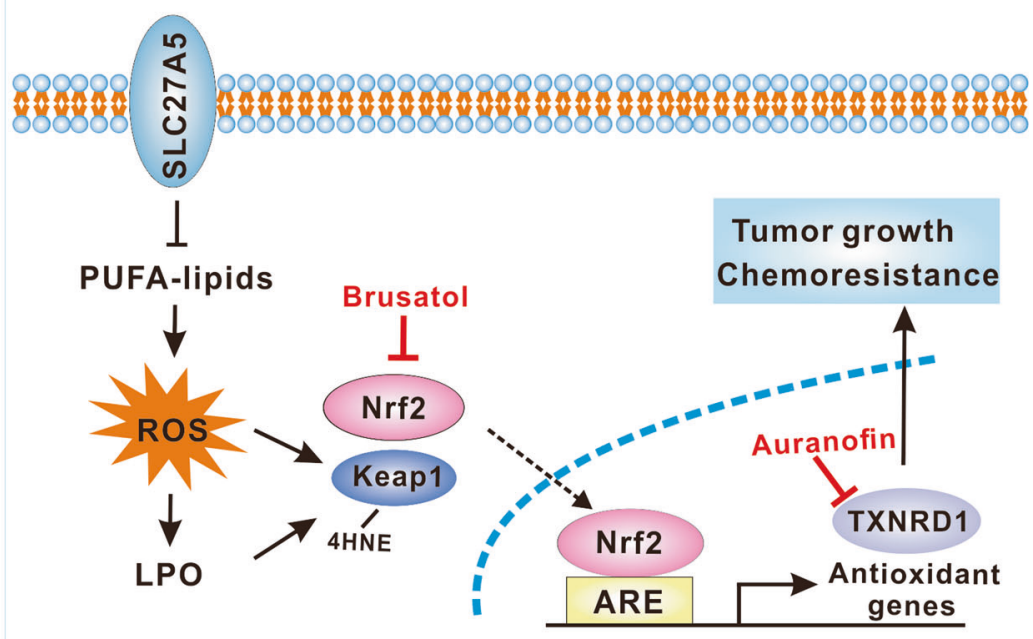


Fig. 7 TXNRD1 knockdown sensitizes hepatoma cells to sorafenib and potentiates apoptosis in vitro and in vivo. TXNRD1 was knocked down in SLC27A5-knockout HepG2 cells using lentiviral shRNA. a, b Sorafenib (Sora) combined with shTXN significantly inhibited cell proliferation $(n=3)$. c Representative images of orthotopic liver implantation of SLC27A5-knockout HepG2 cells with TXNRD1 knockdown $(\operatorname{shTXN})$ in nude mice. d Liver tumor numbers were assessed $(n=6)$. e Ki67 staining in liver tumor tissues $(n=3)$, bar $=$ $50 \mu \mathrm{m}$. f TUNEL staining of apoptotic cells in tumor tissues $(n=3)$, bar $=50 \mu \mathrm{m}$. g Representative fluorescence microscopic images of ROS staining in tumor tissues $(n=3)$. Scale bar: $10 \mu \mathrm{M}$. h Proposed working model of this study. LPO lipid peroxidation. All data are means \pm SDs. $P$ values were derived from repeated-measures ANOVA in $\mathbf{a}$; one-way ANOVA test in $\mathbf{b}, \mathbf{d}$, and $\mathbf{g}(* P<0.05, * * P<0.01$, $* * * P<0.001)$

Acknowledgements We would like to thank Dr T-C He (University of Chicago, USA) for providing the plasmids pAdEasy system, and Prof. Ding Xue (Tsinghua University) for supplying the CRISPR/ Cas9 system. We also thank Prof. Yiguo Zhang (Chongqing University) for providing the pGL3-ARE plasmid. This study was supported by research grants from the China National Natural Science Foundation (grant nos. 81872270 and 81572683 to NT, 81602417 to KW, 81661148057 to AH), the Major National S\&T program (2017ZX10202203-004 to NT), Natural Science Foundation Project of CQ CSTC (grant no. cstc2018jcyjAX0254 to NT), the Program for Innovation Team of Higher Education in Chongqing (grant no. CXTDX201601015), the Leading Talent Program of CQ CSTC (grant no. CSTCCXLJRC201719 to NT), the Scientific Research Innovation Project for Postgraduate in Chongqing and Talent Development Program of CQMU for Postgraduate (grant no. BJRC201705).

Author contributions $\mathrm{AH}, \mathrm{NT}$ and $\mathrm{KW}$ conceived the study and designed the experiments. QZG, GJZ, YQZ and YY performed most experiments and analyzed the data. CC and JX responded to bioinformatics and statistical analysis. LL collected clinical HCC samples. CL generated KEAP1 mutants. YH and XFC assisted with xenograft assays. WLZ assisted with ROS staining. HT and YXC provided guidance and advice. QZG, NT and KW prepared the manuscript with all authors providing feedback.

\section{Compliance with ethical standards}

Conflict of interest The authors declare that they have no conflict of interest.

Publisher's note: Springer Nature remains neutral with regard to jurisdictional claims in published maps and institutional affiliations.

\section{References}

1. Hanahan D, Weinberg RA. Hallmarks of cancer: the next generation. Cell. 2011;144:646-74.

2. Lunt SY, Vander Heiden MG. Aerobic glycolysis: meeting the metabolic requirements of cell proliferation. Annu Rev Cell Dev Biol. 2011;27:441-64.

3. Calvisi DF, Wang C, Ho C, Ladu S, Lee SA, Mattu S, et al. Increased lipogenesis, induced by AKT-mTORC1-RPS6 signaling, promotes development of human hepatocellular carcinoma. Gastroenterology. 2011;140:1071-83.e5.

4. Mashek DG. Hepatic fatty acid trafficking: multiple forks in the road. Adv Nutr Int Rev J. 2013;4:697-710.
5. Anderson CM, Stahl A. SLC27 fatty acid transport proteins. Mol Asp Med. 2013;34:516-28.

6. Doege H, Baillie RA, Ortegon AM, Tsang B, Wu Q, Punreddy S, et al. Targeted deletion of FATP5 reveals multiple functions in liver metabolism: alterations in hepatic lipid homeostasis. Gastroenterology. 2006;130:1245-58.

7. Hubbard B, Doege H, Punreddy S, Wu H, Huang X, Kaushik VK, et al. Mice deleted for fatty acid transport protein 5 have defective bile acid conjugation and are protected from obesity. Gastroenterology. 2006;130:1259-69.

8. Auinger A, Valenti L, Pfeuffer M, Helwig U, Herrmann J, Fracanzani AL, et al. A promoter polymorphism in the liver-specific fatty acid transport protein 5 is associated with features of the metabolic syndrome and steatosis. Horm Metab Res. 2010;42:854-9.

9. Panieri E, Santoro M. ROS homeostasis and metabolism: a dangerous liason in cancer cells. Cell Death Dis. 2016;7:e2253.

10. Gorrini C, Harris IS, Mak TW. Modulation of oxidative stress as an anticancer strategy. Nat Rev Drug Discov. 2013;12:931-47.

11. Ray PD, Huang B-W, Tsuji Y. Reactive oxygen species (ROS) homeostasis and redox regulation in cellular signaling. Cell Signal. 2012;24:981-90.

12. Lu J, Holmgren A. The thioredoxin antioxidant system. Free Radic Biol Med. 2014;66:75-87.

13. Harris IS, Treloar AE, Inoue S, Sasaki M, Gorrini C, Lee KC, et al. Glutathione and thioredoxin antioxidant pathways synergize to drive cancer initiation and progression. Cancer Cell. 2015;27:211-22.

14. Fu B, Meng W, Zeng X, Zhao H, Liu W, Zhang T. TXNRD1 is an unfavorable prognostic factor for patients with hepatocellular carcinoma. BioMed Res Int. 2017;2017:1-8.

15. Yoo M-H, Xu X-M, Carlson BA, Gladyshev VN, Hatfield DL. Thioredoxin reductase 1 deficiency reverses tumor phenotype and tumorigenicity of lung carcinoma cells. J Biol Chem. 2006;281:13005-8.

16. Ungerstedt JS, Sowa Y, Xu W-S, Shao Y, Dokmanovic M, Perez $\mathrm{G}$, et al. Role of thioredoxin in the response of normal and transformed cells to histone deacetylase inhibitors. Proc Natl Acad Sci. 2005;102:673-8.

17. Duan Y, Tian L, Gao Q, Liang L, Zhang W, Yang Y, et al. Chromatin remodeling gene ARID2 targets cyclin D1 and cyclin E1 to suppress hepatoma cell progression. Oncotarget. 2016;7:45863-75.

18. Gao Q, Wang K, Chen K, Liang L, Zheng Y, Zhang Y, et al. HBx protein-mediated ATOH1 downregulation suppresses ARID2 expression and promotes hepatocellular carcinoma. Cancer Sci. 2017;108:1328-37.

19. Ran FA, Hsu PD, Wright J, Agarwala V, Scott DA, Zhang F. Genome engineering using the CRISPR-Cas9 system. Nat Protoc. 2013;8:2281-308.

20. Long M, Rojo de la Vega M, Wen Q, Bharara M, Jiang T, Zhang $\mathrm{R}$, et al. An essential role of NRF2 in diabetic wound healing. Diabetes. 2016;65:780-93.

21. Conner DA. Mouse embryo fibroblast (MEF) feeder cell preparation. Curr Protoc Mol Biol. 2000;51:23.2. 1-23.2.7.

22. Sabharwal SS, Schumacker PT. Mitochondrial ROS in cancer: initiators, amplifiers or an Achilles' heel? Nat Rev Cancer. 2014;14:709-21.

23. Schönfeld P, Wojtczak L. Fatty acids as modulators of the cellular production of reactive oxygen species. Free Radic Biol Med. 2008;45:231-41.

24. Itoh K, Chiba T, Takahashi S, Ishii T, Igarashi K, Katoh Y, et al. An Nrf2/small Maf heterodimer mediates the induction of phase II detoxifying enzyme genes through antioxidant response elements. Biochem Biophys Res Commun. 1997;236:313-22.

25. Hayes JD, Dinkova-Kostova AT. The Nrf2 regulatory network provides an interface between redox and intermediary metabolism. Trends Biochem Sci. 2014;39:199-218. 
26. Levonen AL, Landar A, Ramachandran A, Ceaser EK, Dickinson DA, Zanoni G, et al. Cellular mechanisms of redox cell signalling: role of cysteine modification in controlling antioxidant defences in response to electrophilic lipid oxidation products. Biochem $\mathrm{J}$. 2004;378:373-82.

27. McMahon M, Lamont DJ, Beattie KA, Hayes JD. Keap1 perceives stress via three sensors for the endogenous signaling molecules nitric oxide, zinc, and alkenals. Proc Natl Acad Sci. 2010;107:18838-43.

28. Inami Y, Waguri S, Sakamoto A, Kouno T, Nakada K, Hino O, et al. Persistent activation of Nrf2 through p62 in hepatocellular carcinoma cells. J Cell Biol. 2011;193:275-84.

29. Wang M, Han J, Xing H, Zhang H, Li Z, Liang L, et al. Dysregulated fatty acid metabolism in hepatocellular carcinoma. Hepatic Oncol. 2016;3:241-51.

30. Nath A, Li I, Roberts LR, Chan C. Elevated free fatty acid uptake via CD36 promotes epithelial-mesenchymal transition in hepatocellular carcinoma. Sci Rep. 2015;5:14752.

31. Veglia F, Tyurin VA, Blasi M, De Leo A, Kossenkov AV, Donthireddy L, et al. Fatty acid transport protein 2 reprograms neutrophils in cancer. Nature. 2019;569:73-8.

32. Zhang M, Martino JSD, Bowman RL, Campbell NR, Baksh SC, Simon-Vermot T, et al. Adipocyte-derived lipids mediate melanoma progression via FATP proteins. Cancer Discov. 2018;8:1006-25.

33. Nath A, Chan C. Genetic alterations in fatty acid transport and metabolism genes are associated with metastatic progression and poor prognosis of human cancers. Sci Rep. 2016;6:18669.

34. Knapp P, Chabowski A, Harasiuk D, Górski J. Reversed glucose and fatty acids transporter expression in human endometrial cancer. Horm Metab Res. 2012;44:436-41.

35. Schieber M, Chandel NS. ROS function in redox signaling and oxidative stress. Curr Biol. 2014;24:R453-62.
36. Ogretmen B. Sphingolipid metabolism in cancer signalling and therapy. Nat Rev Cancer. 2017;18:33-50.

37. Lovat PE, Di Sano F, Corazzari M, Fazi B, Donnorso RP, Pearson ADJ, et al. Gangliosides link the acidic sphingomyelinasemediated induction of ceramide to 12-lipoxygenase-dependent apoptosis of neuroblastoma in response to fenretinide. J Natl Cancer Inst. 2004;96:1288-99.

38. Li H, Junk P, Huwiler A, Burkhardt C, Wallerath T, Pfeilschifter $\mathrm{J}$, et al. Dual effect of ceramide on human endothelial cells: induction of oxidative stress and transcriptional upregulation of endothelial nitric oxide synthase. Circulation. 2002;106:2250-6.

39. Alvaro A, Rosales R, Masana L, Vallvé J-C. Polyunsaturated fatty acids down-regulate in vitro expression of the key intestinal cholesterol absorption protein NPC1L1: no effect of monounsaturated nor saturated fatty acids. J Nutr Biochem. 2010;21:518-25.

40. Schulze K. Exome sequencing of hepatocellular carcinomas identifies new mutational signatures and potential therapeutic targets. Nat Genet. 2015;47:505.

41. Luo Y, Eggler AL, Liu D, Liu G, Mesecar AD, van Breemen RB. Sites of alkylation of human Keap1 by natural chemoprevention agents. J Am Soc Mass Spectrom. 2007;18:2226-32.

42. Saito R, Suzuki T, Hiramoto K, Asami S, Naganuma E, Suda H, et al. Characterizations of three major cysteine sensors of Keap1 in stress response. Mol Cell Biol. 2016;36:271-84.

43. Rojo de la Vega M, Chapman E, Zhang DD. NRF2 and the hallmarks of cancer. Cancer Cell. 2018;34:21-43.

44. Eriksson SE, Prast-Nielsen S, Flaberg E, Szekely L, Arnér ESJ. High levels of thioredoxin reductase 1 modulate drug-specific cytotoxic efficacy. Free Radic Biol Med. 2009;47:1661-71.

45. Hu J, Zhang H, Cao M, Wang L, Wu S, Fang B. Auranofin enhances Ibrutinib's anticancer activity in EGFR-mutant lung adenocarcinoma. Mol Cancer Ther. 2018;17:2156-63. 